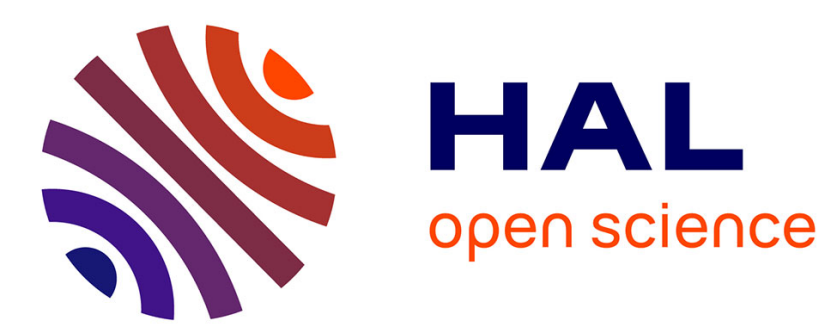

\title{
Finite-time observer-based output-feedback control for the global stabilisation of the PVTOL aircraft with bounded inputs
}

\author{
Arturo Zavala-Río, Isabelle Fantoni, Guillaume Sanahuja
}

\section{To cite this version:}

Arturo Zavala-Río, Isabelle Fantoni, Guillaume Sanahuja. Finite-time observer-based output-feedback control for the global stabilisation of the PVTOL aircraft with bounded inputs. International Journal of Systems Science, 2016, 47 (7), pp.1543-1562. 10.1080/00207721.2014.938906 . hal-01137636

\author{
HAL Id: hal-01137636 \\ https://hal.science/hal-01137636
}

Submitted on 28 Jul 2015

HAL is a multi-disciplinary open access archive for the deposit and dissemination of scientific research documents, whether they are published or not. The documents may come from teaching and research institutions in France or abroad, or from public or private research centers.
L'archive ouverte pluridisciplinaire HAL, est destinée au dépôt et à la diffusion de documents scientifiques de niveau recherche, publiés ou non, émanant des établissements d'enseignement et de recherche français ou étrangers, des laboratoires publics ou privés. 


\title{
Finite-time-observer-based output-feedback control for the global stabilization of the PVTOL aircraft with bounded inputs
}

\author{
A. Zavala-Río ${ }^{a *}$, I. Fantoni ${ }^{b}$ and G. Sanahuja ${ }^{b}$ \\ a IPICYT, Camino a la Presa San José 2055, Lomas 4a. Sección 78216, San Luis Potosí, S.L.P., Mexico; \\ ${ }^{b}$ Université de Technologie de Compiègne - CNRS, UMR 7253 Heudiasyc, Rue Roger Couttolenc, CS 60319, \\ 60200 Compiègne, France
}

\begin{abstract}
In this work, an output-feedback scheme for the global stabilization of the PVTOL aircraft with bounded inputs is developed taking into account the positive nature of the thrust. The global stabilization objective is proven to be achieved avoiding input saturation and by exclusively considering the system positions in the feedback. To cope with the lack of velocity measurements, the proposed algorithm involves a finite-time observer. The generalized versions of the involved finite-time stabilizers have not only permitted to solve the output-feedback stabilization problem avoiding input saturation, but also provide additional flexibility in the control design that may be used in aid of performance improvements. With respect to previous approaches, the developed finite-time-observer-based scheme guarantees the global stabilization objective disregarding velocity measurements in a bounded input context. Simulation tests corroborate the analytical developments. The study includes further experimental results on an actual flying device.
\end{abstract}

Index Terms

non-linear control; global stabilization; output feedback; finite-time observers; bounded inputs; PVTOL aircraft

\section{INTRODUCTION}

Vertical/short take-off and landing (V/STOL) are capabilities of certain type of aircraft designed for particular strategic purposes. This is the case, for instance, of the YAV-8B Harrier (McDonnell Aircraft Company) [Hauser et al.(1992)], [Chemori and Marchand (2008)], [Wang et al.(2008)] (a long list of V/STOL aircraft can be found for instance in

[Saeed and Gratton (2010)]). The development of control algorithms dealing with the basic dynamic features of such type of physical systems could hardly be carried out under the simultaneous consideration of the whole set of involved phenomena, as pointed out for instance in [Hauser et al.(1992), §2.1]. For this reason, a prototype of a Planar Vertical Take-off and Landing (PVTOL) aircraft has been introduced in [Hauser et al.(1992)], where it is claimed to model the natural restriction of a V/STOL aircraft to jet-borne operation (e.g. hover) in a vertical-lateral plane. Such a prototype has a particular well-posed dynamic model (to be presented in Section II) whose complexities have attracted numerous researchers, becoming a benchmark in the study on the control of underactuated systems [Liu and Yu (2013)], [Fantoni and Lozano (2002)].

The design of a suitable control algorithm for a PVTOL aircraft has proven to constitute a challenging task. This is mainly due to the complexities characterizing its dynamic model: it is highly nonlinear, underactuated, and has a signed input (unidirectional thrust). The efforts devoted to such a particular study case have given rise to diverse approaches. For instance, in view of the unstable non-zero dynamics obtained through the application of conventional geometric control techniques, an approximate input-output design procedure dealing with non-minimum phase nonlinear systems has been proposed in [Hauser et al.(1992)]. Through the definition of a suitable output variable that renders the PVTOL aircraft dynamics a flat system, a scheme for output tracking has been developed in [Martin et al.(1996)]. By means of a suitable problem formulation, an optimal control technique is applied in [Lin et al.(1999)]. Based on a reference model and the consideration of particular variable transformations, global uniformly ultimately bounded tracking is achieved in [Setlur et al.(2001)]. An error feedback dynamic controller being robust with respect to parametric uncertainties was proposed in [Marconi et al.(2002)] for autonomous vertical landing on oscillating platforms. Applying a decoupling change of coordinates, global stabilization was proven to be achieved through backstepping under the consideration of input coupling (generally neglected) in [Olfati-Saber (2002)]. A regulation algorithm that uses the thrust to force an approximately linear vertical motion behavior and the torque input to control the rest of the system variables was presented in [Lozano et al.(2004)]. A globally stabilizing nonlinear feedback control law that casts the system

${ }^{*}$ Corresponding author. Email: azavala@ipicyt.edu.mx 
into a cascade structure was proposed in [Wood and Cazzolato (2007)]. By transforming the system dynamics into a chain of integrators with nonlinear perturbations, global stabilization was also proven to be achieved in [Ye et al.(2007)] through a control technique that involves saturation functions. An open-loop exact tracking scheme ensuring bounded internal dynamics was developed in [Consolini and Tosques (2007)] through a Poincaré map approach. Based on partial feedback linearization and optimal trajectory generation to enhance the behavior and the stability of the system internal dynamics, a nonlinear predictionbased stabilization algorithm was proposed in [Chemori and Marchand (2008)]. Through a geometrical control approach, output tracking was achieved in [Wang et al.(2008)]. A path following controller with the properties of output invariance of the path and boundedness of the roll dynamics was proposed in [Consolini et al.(2010)]. Alternative stabilization approaches based on non-embedded saturation functions were presented in [Sanahuja et al.(2010)] disregarding lateral coupling effects. Output tracking with input disturbance rejection is achieved through the method developed in [Su and Lin (2011)]. Further stabilization results were achieved through Lyapunov-based design in [Turker, Gorgun and Canserver (2012)] for the uncoupled dynamics, and in [Turker, Olfaz, Gorgun and Canserver (2012)] under the consideration of the whole dynamics.

Other works have considered additional constraints that commonly arise in real applications. For instance, state-feedback approaches have been developed in [Zavala-Río et al.(2003)], [Lopez-Araujo et al.(2010)], [Ailon (2010)] under the consideration of bounded inputs: in [Zavala-Río et al.(2003)], global stabilization was achieved neglecting the lateral force coupling through the use of embedded (linear) saturation functions; this approach was further proven to achieve the global stabilization objective under the additional consideration of the lateral force coupling in [Lopez-Araujo et al.(2010)] (for sufficiently small values of the parameter characterizing such a coupling); in [Ailon (2010)], a semiglobal tracking controller was developed involving smooth sigmoidal functions. Furthermore, schemes that achieve the control objective through output feedback have been proposed in [Do et al.(2003)], [Wang et al.(2009)], [Su and Lin (2013)], [Frye et al.(2010)], [Wang et al.(2010)]: design and analysis procedures were developed disregarding velocity measurements in [Do et al.(2003)] for tracking, [Wang et al.(2009)] and [Su and Lin (2013)] for tracking with delayed outputs, and [Frye et al.(2010)] for stabilization, under the consideration of Luenberger-type, two-step structured, full-order, and finite-time observers respectively; furthermore, a tracking controller that avoids angular motion (position and velocity) variables in the feedback is presented in [Wang et al.(2010)] based on linear observer and sliding mode techniques. However, these output-feedback approaches were developed disregarding input constraints.

Inspired by the techniques involved in [Frye et al.(2010)], this work proposes an output-feedback control scheme for the global stabilization of the PVTOL aircraft, taking into account the unidirectional nature of the thrust, under the consideration of bounded inputs. First, a state-feedback algorithm is presented and proven to globally stabilize the closed-loop system avoiding input saturation. Then, the same algorithm is proven to achieve the global stabilization objective — avoiding input saturationby replacing the velocity variables by auxiliary states coming from a finite-time observer, which is analytically proven (under ideal conditions) to exactly reproduce the aircraft positions and velocities after a finite-time transient during which the system variables remain bounded. The finite-time stabilizers considered in this work are generalized versions of those involved in [Frye et al.(2010)]. Such a generalized structure gives rise to an additional degree of design flexibility that has not only permitted to solve the output-feedback stabilization problem in a bounded input context, but may also be used in aid of performance improvements. Simulation tests corroborate the efficiency of the proposed scheme. Furthermore, experimental results on an actual flying device are additionally included. It is worth pointing out that experimental corroborations of algorithms designed for the control of the PVTOL aircraft are scarcely included in the literature. For instance, from all the above cited works only those in [Lozano et al.(2004)], [Sanahuja et al.(2010)], [Lopez-Araujo et al.(2010)] include experimental tests. Such works have thus corroborated the implementability of their proposed schemes despite the evident effects of modelling and technical imprecisions.

The paper is organized as follows. Section II presents the PVTOL aircraft dynamics. Section III states the notation, definitions, and results used to develop the proposed result. Sections IV and V respectively present the proposed state- and output-feedback stabilization schemes. Simulation and experimental results are respectively shown in Sections VI and VII. Finally, conclusions are given in Section VIII.

\section{THE PVTOL AIRCRAFT DYNAMICS}

The PVTOL aircraft dynamics is given by the following equations [Hauser et al.(1992)]:

$$
\begin{aligned}
& \ddot{\xi}=-u_{1} \sin \theta+\varepsilon u_{2} \cos \theta \\
& \ddot{\zeta}=u_{1} \cos \theta+\varepsilon u_{2} \sin \theta-1 \\
& \ddot{\theta}=u_{2}
\end{aligned}
$$

where $\xi$ and $\zeta$ respectively denote the center of mass horizontal and vertical positions, and $\theta$ is the roll angle of the aircraft with the horizon. The control inputs $u_{1}$ and $u_{2}$ are respectively the thrust — non-negative by nature [Hauser et al.(1992)] and the rolling moment. The constant "-1" is the normalized gravitational acceleration. The parameter $\varepsilon$ is a coefficient characterizing the coupling between the rolling moment and the lateral acceleration of the aircraft. Its value is generally so 
small that it is often neglected (see for instance [Hauser et al.(1992), §2.4]). However, provided that its value is available, the following translational coordinate transformation [Olfati-Saber (2002)]

$$
\begin{aligned}
& x=\xi-\xi_{0}-\varepsilon \sin \theta \\
& y=\zeta-\zeta_{0}+\varepsilon(\cos \theta-1)
\end{aligned}
$$

with $\xi_{0}$ and $\zeta_{0}$ respectively being horizontal and vertical positions mapped to the origin of the transformed coordinate space, and input variable redefinition in terms of an auxiliary input variable $\bar{u}_{1}$

$$
u_{1}=\bar{u}_{1}+\varepsilon \dot{\theta}^{2}
$$

give rise to the following $\varepsilon$-free equivalent dynamics

$$
\begin{aligned}
& \ddot{x}=-\bar{u}_{1} \sin \theta \\
& \ddot{y}=\bar{u}_{1} \cos \theta-1 \\
& \ddot{\theta}=u_{2}
\end{aligned}
$$

Under the consideration of bounded inputs, i.e. $0 \leq u_{1} \leq U_{1}$ and $\left|u_{2}\right| \leq U_{2}$ for some constants ${ }^{1} U_{1}>1$ and $U_{2}>0$, we state the control objective as being the global stabilization of the solution $(\xi, \zeta, \theta)(t) \equiv\left(\xi_{d}, \zeta_{d}, 0\right)$ of system (1), for any constant desired horizontal and vertical positions $\xi_{d}$ and $\zeta_{d}$ respectively, through a bounded control scheme that only feeds back configuration variables and avoids input saturation i.e. such that $0<u_{1}(t)<U_{1}$ and $\left|u_{2}(t)\right|<U_{2}, \forall t \geq 0$.

Remark 1. Note from Eqs. (2) with $\left(\xi_{0}, \zeta_{0}\right)=\left(\xi_{d}, \zeta_{d}\right)$ that $(\xi, \zeta, \theta)=\left(\xi_{d}, \zeta_{d}, 0\right) \Longleftrightarrow(x, y, \theta)=(0,0,0)$, in view of which the control objective can be equivalently achieved through an analog global stabilization of the trivial solution $(x, y, \theta)(t) \equiv(0,0,0)$ of system (4), as long as the designed control law guarantees that $\bar{u}_{1}$ can be suitably bounded and (3) ultimately holds.

\section{PRELIMINARIES}

The following notation is used throughout the paper. Let $\mathbb{N}$ be the set of natural numbers and $\mathbb{Z}^{+}$stand for the set of nonnegative integer numbers. For particular values $m \in \mathbb{N}$ and $n \in \mathbb{Z}^{+}, \mathbb{N}_{m}$ and $\mathbb{Z}_{n}^{+}$will represent the subsets of natural numbers up to $m$ and nonnegative integer numbers up to $n$ respectively, i.e. $\mathbb{N}_{m}=\{1, \ldots, m\}$ and $\mathbb{Z}_{n}^{+}=\{0, \ldots, n\}$. Let $0_{n}$ stand for the origin of $\mathbb{R}^{n}$. For any $x \in \mathbb{R}^{n}, x_{i}$ represents its $i^{\text {th }}$ element, while $\|\cdot\|$ is used to denote the standard Euclidean vector norm, i.e. $\|x\|=\left[\sum_{i=1}^{n} x_{i}^{2}\right]^{1 / 2}$. Let $\mathbb{R}_{>0}^{n} \triangleq\left\{x \in \mathbb{R}^{n}: x_{i}>0, \forall i \in \mathbb{N}_{n}\right\}$ and $\mathbb{R}_{>0}^{n} \triangleq\left\{x \in \mathbb{R}^{n}: x_{i} \geq 0, \forall i \in \mathbb{N}_{n}\right\}$. Let $\mathscr{A}$ and $\mathscr{E}$ be subsets (with nonempty interior) of some vector spaces $\mathbb{A}$ and $\mathbb{E}$ respectively. The image of $\mathscr{B} \subset \mathscr{A}$ under $v: \mathscr{A} \rightarrow \mathscr{E}$ is denoted $v(\mathscr{B})$. As conventionally, the inverse of an invertible function $v$ is expressed as $v^{-1}$. We denote $\mathscr{C}^{m}(\mathscr{A} ; \mathscr{E})$ the set of $m$-times continuously differentiable functions from $\mathscr{A}$ to $\mathscr{E}$, with $\mathscr{C}^{0}$ the set of continuous functions. Consider a scalar function $\zeta \in \mathscr{C}^{m}(\mathbb{R} ; \mathbb{R})$ with $m \in \mathbb{Z}^{+}$. The following notation will be used: $\zeta^{\prime}: s \mapsto \frac{d}{d s} \zeta$, when $m \geq 1 ; \zeta^{\prime \prime}: s \mapsto \frac{d^{2}}{d s^{2}} \zeta$, when $m \geq 2$; and more generally $\zeta^{(n)}: s \mapsto \frac{d^{n}}{d s^{n}} \zeta, \forall n \in \mathbb{N}_{m}$, and $\zeta^{(0)}=\zeta$. We denote sat $(s)$ the standard (unitary) saturation function, i.e. $\operatorname{sat}(s)=\operatorname{sign}(s) \min \{|s|, 1\}$. In the rest of this section, some definitions and results that underlie the contribution of this work are stated.

Definition 1. [Hong (2002)], [Bacciotti and Rosier (2005)], [Bhat and Bernstein (2005)], [Aeyels and de Leenheer (2002)] $A$ family of dilations $\delta_{\varepsilon}^{r}$ is defined as $\delta_{\varepsilon}^{r}(x)=\left(\varepsilon^{r_{1}} x_{1}, \ldots, \varepsilon^{r_{n}} x_{n}\right), \forall x \in \mathbb{R}^{n}, \forall \varepsilon>0$, where $r=\left(r_{1}, \ldots, r_{n}\right)$, with the dilation coefficients $r_{1}, \ldots, r_{n}$ being positive real numbers. A function $V: \mathbb{R}^{n} \rightarrow \mathbb{R}$, resp. vector field $f: \mathbb{R}^{n} \rightarrow \mathbb{R}^{n}$, is homogeneous of degree $\alpha$ with respect to the family of dilations $\delta_{\varepsilon}^{r}$ —or equivalently, it is said to be $r$-homogeneous of degree $\alpha-$ if

$$
V\left(\delta_{\varepsilon}^{r}(x)\right)=\varepsilon^{\alpha} V(x)
$$

resp.

$$
f\left(\delta_{\varepsilon}^{r}(x)\right)=\varepsilon^{\alpha} \delta_{\varepsilon}^{r}(f(x))
$$

$\forall x \in \mathbb{R}^{n}, \forall \varepsilon>0$.

Definition 2. [Zavala-Río and Fantoni (2014)] Given $r \in \mathbb{R}_{>0}^{n}$, a neighborhood of the origin $D \subset \mathbb{R}$ is said to be $\delta_{\varepsilon}^{r}$-connected if, for every $x \in D, \delta_{\varepsilon}^{r}(x) \in D$ for all $\varepsilon \in(0,1)$. A function $V: \mathbb{R}^{n} \rightarrow \mathbb{R}$, resp. vector field $f: \mathbb{R}^{n} \rightarrow \mathbb{R}^{n}$, is locally homogeneous of degree $\alpha$ with respect to the family of dilations $\delta_{\varepsilon}^{r}$ - or equivalently, it is said to be locally $r$-homogeneous of degree $\alpha$ if there exists a $\delta_{\varepsilon}^{r}$-connected open neighborhood of the origin $D \subset \mathbb{R}^{n}$-referred to as the domain of homogeneity- such that Eq. (5), resp. (6), is satisfied for every $x \in D$ and all $\varepsilon \in \mathbb{R}_{>0}$ such that $\delta_{\varepsilon}^{r}(x) \in D$.

Observe that a locally $r$-homogeneous function or vector field with domain of homogeneity $D \subset \mathbb{R}^{n}$ is not necessarily $r$ homogeneous, unless $D=\mathbb{R}^{n}$, while an $r$-homogeneous function or vector field is locally $r$-homogeneous for any $\delta_{\varepsilon}^{r}$-connected

\footnotetext{
${ }^{1}$ As pointed out in [Zavala-Río et al.(2003)] and [Lopez-Araujo et al.(2010)], notice from the vertical motion equation (1b) that $U_{1}>1$ is a necessary condition for the system in Eqs. (4) to be stabilizable, since any steady-state condition implies that the aircraft weight be compensated.
} 
open neighborhood of the origin $D \subset \mathbb{R}^{n}$. Let us further notice that the degree of homogeneity $\alpha$ of a function $V$ or a vector field $f$ is generally determined by the (vector) value of $r$ through which Definition 1 or 2 is satisfied. In other words, $\alpha$ in Definitions 1 and 2 is generally a function of $r$.

Definition 3. [Hong (2002)], [Bacciotti and Rosier (2005)], [Bhat and Bernstein (2005)], [Bhat and Bernstein (2000)] Consider an $n$-th order autonomous system

$$
\dot{x}=f(x)
$$

where $f: \mathscr{D} \rightarrow \mathbb{R}^{n}$ is continuous on an open neighborhood $\mathscr{D} \subset \mathbb{R}^{n}$ of the origin and $f\left(0_{n}\right)=0_{n}$, and let $x\left(t ; x_{0}\right)$ represent the system solution with initial condition $x\left(0 ; x_{0}\right)=x_{0}$. The origin is said to be a finite-time stable equilibrium of system (7) if it is Lyapunov stable and there exist an open neighborhood $\mathscr{N} \subset \mathscr{D}$ being positively invariant with respect to (7), and a positive definite function $T: \mathscr{N} \rightarrow \mathbb{R}_{>0}$, called the settling-time function, such that $x\left(t ; x_{0}\right) \neq 0_{n}, \forall t \in\left[0, T\left(x_{0}\right)\right), \forall x_{0} \in \mathscr{N} \backslash\left\{0_{n}\right\}$, and $x\left(t ; x_{0}\right)=0_{n}, \forall t \geq T\left(x_{0}\right), \forall x_{0} \in \mathscr{N}$. The origin is said to be a globally finite-time stable equilibrium of system (7) if it is finite-time stable with $\mathscr{N}=\mathscr{D}=\mathbb{R}^{n}$.

Theorem 1. [Zavala-Río and Fantoni (2014)] Consider system (7) with $\mathscr{D}=\mathbb{R}^{n}$. Suppose that $f$ is a locally $r$-homogeneous vector field of degree $k$ with domain of homogeneity $D \subset \mathbb{R}^{n}$. Then, the origin is a globally finite-time stable equilibrium of system (7) if and only if it is globally asymptotically stable and $k<0$.

The proof of Theorem 1 has been thoroughly developed in [Zavala-Río and Fantoni (2014)]. A partial version - namely, the sufficiency implication - of this theorem was used in [Frye et al.(2010)] to support the result presented therein.

Definition 4. 1) A continuous scalar function $\sigma: \mathbb{R} \rightarrow \mathbb{R}$ will be said to be:

a) bounded by $M$ if $|\sigma(s)| \leq M, \forall s \in \mathbb{R}$, for some positive constant $M$;

b) strictly passive if $s \sigma(s)>0, \forall s \neq 0$;

c) strongly passive if it is a strictly passive function satisfying

$$
|\sigma(s)| \geq \kappa|a \operatorname{sat}(s / a)|^{\alpha}=\kappa(\min \{|s|, a\})^{\alpha}
$$

$\forall s \in \mathbb{R}$, for some positive constants $\kappa, \alpha$, and a.

2) A nondecreasing strictly passive function being bounded by $M$, locally $r$-homogeneous of degree $\alpha>0$ for some $r>0$, and locally Lipschitz-continuous on $\mathbb{R} \backslash\{0\}$, will be said to be a homogeneous saturation (function) for $(\alpha, r, M)$.

3) A nondecreasing Lipschitz-continuous strictly passive function being bounded by $M$ will be said to be $a$ generalized saturation (function) with bound $M$.

4) A generalized saturation function, $\sigma$, with bound $M$ is said to be a linear saturation (function) for (L,M) if there is a positive constant $L \leq M$ such that $\sigma(s)=s, \forall|s| \leq L$ [Teel (1992)].

For a generalized or homogeneous saturation function $\sigma(s), M^{+} \triangleq \lim _{s \rightarrow \infty} \sigma(s)$ and $M^{-} \triangleq-\lim _{s \rightarrow-\infty} \sigma(s)$, which are called the limit bounds of $\sigma$, while $\bar{M} \triangleq \max \left\{M^{+}, M^{-}\right\}$and $\underline{M} \triangleq \min \left\{M^{+}, M^{-}\right\}$.

Observe that $M \leq \bar{M} \leq M$, i.e. $M^{+}$and $M^{-}$does not necessarily have the same value (but could be different), and $M$ is not necessarily equal to $\bar{M}$ (but could be greater).

Remark 2. Let us note that a nondecreasing strictly passive function $\sigma$ is strongly passive. Indeed, notice that the strictly passive character of $\sigma$ implies the existence of a sufficiently small $a>0$ such that $|\sigma(s)| \geq \kappa|s|^{\alpha}, \forall|s| \leq a$, for some positive constants $\kappa$ and $\alpha$, while from its non-decreasing character we have that $|\sigma(s)| \geq|\sigma(\operatorname{sign}(s) a)| \geq \kappa a^{\alpha}, \forall|s| \geq a$, and thus $|\sigma(s)| \geq \kappa(\min \{|s|, a\})^{\alpha}=\kappa|a \operatorname{sat}(s / a)|^{\alpha}, \forall s \in \mathbb{R}$.

Lemma 1. Let $\sigma \in \mathscr{C}^{m}(\mathbb{R} ; \mathbb{R})$, for some $m \in \mathbb{N}$, be a generalized saturation function with bound $M$. Then:

1) $\lim _{|s| \rightarrow \infty} s^{p} \sigma^{(q)}(s)=0$ for all $p \in \mathbb{Z}^{+}$and all $q \in \mathbb{N}_{m}$;

2) for all $p \in \mathbb{Z}^{+}$and all $q \in \mathbb{N}_{m}$, there exist $A^{p, q} \in(0, \infty)$ such that $\left|s^{p} \sigma^{(q)}(s)\right| \leq A^{p, q}, \forall s \in \mathbb{R}$.

Proof: See Appendix A.

Lemma 2. Let $\sigma: \mathbb{R} \rightarrow \mathbb{R}, \sigma_{1}: \mathbb{R} \rightarrow \mathbb{R}$, and $\sigma_{2}: \mathbb{R} \rightarrow \mathbb{R}$ be strongly passive functions and $k$ be a positive constant. Then:

1) $\int_{0}^{s} \sigma(k v) d v>0, \forall s \neq 0$;

2) $\int_{0}^{s} \sigma(k v) d v \rightarrow \infty$ as $|s| \rightarrow \infty$;

3) $\sigma_{1} \circ \sigma_{2}$ is strongly passive.

Proof: See Appendix B.

Lemma 3. Let $\sigma_{0}: \mathbb{R} \rightarrow \mathbb{R}$ be a strictly increasing function, $\sigma_{1}: \mathbb{R} \rightarrow \mathbb{R}$ be strictly passive, and $k$ be a positive constant. Then: $s_{1}\left[\sigma_{0}\left(\sigma_{1}\left(k s_{1}\right)+s_{2}\right)-\sigma_{0}\left(s_{2}\right)\right]>0, \forall s_{1} \neq 0, \forall s_{2} \in \mathbb{R}$.

Proof: See Appendix C. 
Lemma 4. Consider the second-order system

$$
\begin{array}{ll}
\dot{x}_{1}=x_{2} & \triangleq f_{1}\left(x_{1}, x_{2}\right) \\
\dot{x}_{2}=-\sigma_{3}\left(\sigma_{1}\left(k_{1} x_{1}\right)+\sigma_{2}\left(k_{2} x_{2}\right)\right) & \triangleq f_{2}\left(x_{1}, x_{2}\right)
\end{array}
$$

where $\sigma_{3}: \mathbb{R} \rightarrow \mathbb{R}$ is a strictly increasing strictly passive function, $\sigma_{1}: \mathbb{R} \rightarrow \mathbb{R}$ is strongly passive, and $\sigma_{2}: \mathbb{R} \rightarrow \mathbb{R}$ is strictly passive, all three being locally Lipschitz on $\mathbb{R} \backslash\{0\}$, and $k_{1}$ and $k_{2}$ are (arbitrary) positive constants. For this dynamical system, $(0,0)$ is a globally asymptotically stable equilibrium. If in addition, for every $i \in \mathbb{N}_{2}, \sigma_{i}(s)$ is locally $r_{i}$-homogeneous of degree $\alpha$ with domain of homogeneity $D_{i} \triangleq\left\{s \in \mathbb{R}:|s|<\rho_{i} \in(0, \infty]\right\}$, and $\sigma_{3}(s)$ is locally $\alpha$-homogeneous of degree $\alpha_{3}$ with domain of homogeneity $D_{3} \triangleq\left\{s \in \mathbb{R}:|s|<\rho_{3} \in(0, \infty]\right\}$, for some dilation coefficients such that

$$
\alpha_{3}=2 r_{2}-r_{1}>0>r_{2}-r_{1}
$$

then $(0,0)$ is globally finite-time stable.

Proof: See Appendix D.

Corollary 1. For every $i \in \mathbb{N}_{3}$, let $\sigma_{i}(s)=\kappa_{i} \operatorname{sign}(s)|s|{ }_{i}, \forall|s|<\rho_{i} \in(0, \infty]$, with $\kappa_{i}$ and $\beta_{i}$ being positive constants. Thus, for any $\left(r_{1}, r_{2}\right) \in \mathbb{R}_{>0}^{2}$ such that $r_{1} \beta_{1}=r_{2} \beta_{2}=\alpha, \sigma_{j}(s)$ is locally $r_{j}$-homogeneous of degree $\alpha$ with domain of homogeneity $D_{j}=\left\{s \in \mathbb{R}:|s|<\rho_{j}\right\}$, for every $j \in \mathbb{N}_{2}$, and $\sigma_{3}(s)$ is $\alpha$-homogeneous of degree $\alpha_{3}$ with domain of homogeneity $D_{3}=\{s \in$ $\left.\mathbb{R}:|s|<\rho_{3}\right\}$, guaranteeing the satisfaction of (9), if and only if

$$
\beta_{3}=\frac{2}{\beta_{2}}-\frac{1}{\beta_{1}}>0>\frac{1}{\beta_{2}}-\frac{1}{\beta_{1}}
$$

Proof: See Appendix E.

Remark 3. Let us note, from Lemma 4 and Corollary 1 , that for system (8) with a strictly increasing strictly passive $\sigma_{3}(s)$, a strongly passive $\sigma_{1}(s)$, and a strictly passive $\sigma_{2}(s)$, all three being locally Lipschitz-continuous on $\mathbb{R} \backslash\{0\}$, such that, for every $i \in \mathbb{N}_{3}, \sigma_{i}(s)=\kappa_{i} \operatorname{sign}(s)|s|^{\beta_{i}}, \forall|s|<\rho_{i} \in(0, \infty]$, with $\kappa_{i}>0$ and positive values of $\beta_{i}$ satisfying $(10)$, $(0,0)$ is a globally finite-time stable equilibrium. In particular, for the special case generated by taking $\sigma_{3}(s)=s, \forall s \in \mathbb{R}$, and, for every $i \in \mathbb{N}_{2}$, $\sigma_{i}(s)=k_{i} \operatorname{sign}(s) \max \left\{|s|^{\beta_{i}},|s|\right\}, \forall s \in \mathbb{R}$, under the satisfaction of (10) with $\beta_{3}=1$-equivalently expressed as: $0<\beta_{1}<1$ and $\beta_{2}=2 \beta_{1} /\left(1+\beta_{1}\right)-$ global finite-time stability of the origin was stated in [Frye et al.(2010), Lemma 2.2]. Furthermore, for the particular case obtained by defining $\sigma_{3}(s)=s, \forall s \in \mathbb{R}$, and, for every $i \in \mathbb{N}_{2}, \sigma_{i}(s)=\operatorname{sign}(s)|s| \beta_{i}, \forall s \in \mathbb{R}$, with positive values of $\beta_{i}$ satisfying (10) with $\beta_{3}=1$ as well, finite-time stability of the origin was previously proved in [Bacciotti and Rosier (2005), Example 5.6]. Interesting enough, the dynamical system generated in this latter special case was previously considered in [Haimo (1986), Corollary 1] where, by means of a different analysis — which does not take into account the homogeneity properties of the involved functions-, finite-time stability of the trivial solution was proven to take place provided that $0<\beta_{1}<1$ and $0<\beta_{2}<2 \beta_{1} /\left(1+\beta_{1}\right)$.

Lemma 5. Consider the second-order system

$$
\begin{aligned}
& \dot{x}_{1}=x_{2}-\sigma_{1}\left(k_{1} x_{1}\right) \triangleq f_{1}\left(x_{1}, x_{2}\right) \\
& \dot{x}_{2}=-\sigma_{2}\left(k_{2} x_{1}\right) \triangleq f_{2}\left(x_{1}, x_{2}\right)
\end{aligned}
$$

where $\sigma_{1}: \mathbb{R} \rightarrow \mathbb{R}$ is a strictly passive function and $\sigma_{2}: \mathbb{R} \rightarrow \mathbb{R}$ is strongly passive, both being locally Lipschitz on $\mathbb{R} \backslash\{0\}$, and $k_{1}$ and $k_{2}$ are (arbitrary) positive constants. For this dynamical system, $(0,0)$ is a globally asymptotically stable equilibrium. If in addition, for every $i \in \mathbb{N}_{2}, \sigma_{i}(s)$ is locally $r_{0}$-homogeneous of degree $\alpha_{i}$, with domain of homogeneity $D_{i}=\{s \in \mathbb{R}:|s|<$ $\left.\rho_{i} \in(0, \infty]\right\}$, for some (common) dilation coefficient such that

$$
\alpha_{2}=2 \alpha_{1}-r_{0}>0>\alpha_{1}-r_{0}
$$

then $(0,0)$ is globally finite-time stable.

Proof: See Appendix F.

Corollary 2. For every $i \in \mathbb{N}_{2}$, let $\sigma_{i}(s)=\kappa_{i} \operatorname{sign}(s)|s| \beta_{i}, \forall|s|<\rho_{i} \in(0, \infty]$, with $\kappa_{i}$ and $\beta_{i}$ being positive constants. Thus, $\sigma_{i}(s)$, $i=1,2$, are locally $r_{0}$-homogeneous of degree $\alpha_{i}$, for some (common) dilation coefficient $r_{0}$ such that (12) is satisfied if and only if

$$
\beta_{2}=2 \beta_{1}-1>0>\beta_{1}-1
$$

Proof: See Appendix G.

Remark 4. Let us note, from Lemma 5 and Corollary 2, that for system (11) with strictly passive $\sigma_{1}(s)$ and strongly passive $\sigma_{2}(s)$, both being locally Lipschitz-continuous on $\mathbb{R} \backslash\{0\}$, such that, for every $i \in \mathbb{N}_{2}, \sigma_{i}(s)=\kappa_{i} \operatorname{sign}(s)|s| \beta_{i}, \forall|s|<\rho_{i} \in(0, \infty]$, with $\kappa_{i}>0$ and positive values of $\beta_{i}$ satisfying (13) - equivalently expressed as: $0.5<\beta_{1}<1$ and $\beta_{2}=2 \beta_{1}-1-$, $(0,0)$ 
is a globally finite-time stable equilibrium. In particular, for the special case of system (11) generated by taking $\sigma_{i}(s)=$ $k_{i} \operatorname{sign}(s) \max \left\{|s|^{\beta_{i}},|s|\right\}, \forall s \in \mathbb{R}, i=1,2$, with positive values of $\beta_{i}$ satisfying (13), global finite-time stability of the origin was stated in [Frye et al.(2010), Lemma 2.3]. Furthermore, for the alternative case obtained by defining $\sigma_{i}(s)=k_{i} \operatorname{sign}(s)|s|^{\beta_{i}}$, $\forall s \in \mathbb{R}, i=1,2$, with positive values of $\beta_{i}$ satisfying (13) as well, global finite-time stability of the origin was previously stated in [Hong et al.(2001), Proposition 1].

\section{STATE-FEEDBACK GLOBAL STABILIZER}

We define the following state-feedback controller

$$
\begin{gathered}
u_{1}=\bar{u}_{1}+\varepsilon\left[\sigma_{0}(\dot{\theta})\right]^{2} \\
\bar{u}_{1}=\sqrt{v_{1}^{2}+\left(1+v_{2}\right)^{2}} \\
v_{1}=-k_{0} \sigma_{12}\left(k_{12} \dot{x}+\sigma_{11}\left(k_{11} x\right)\right) \\
v_{2}=-\sigma_{22}\left(k_{22} \dot{y}+\sigma_{21}\left(k_{21} y\right)\right) \\
u_{2}=\sigma_{30}\left(\ddot{\theta}_{d}\right)-\sigma_{33}\left(\sigma_{31}\left(k_{31}\left(\theta-\theta_{d}\right)\right)+\sigma_{32}\left(k_{32}\left(\dot{\theta}-\dot{\theta}_{d}\right)\right)\right) \\
\theta_{d}=\arctan \left(-v_{1}, 1+v_{2}\right)
\end{gathered}
$$

where (based on the variable relations stated through Eqs. (2))

$$
\begin{gathered}
x=\xi-\xi_{d}-\varepsilon \sin \theta \\
\dot{x}=\dot{\xi}-\varepsilon \dot{\theta} \cos \theta \\
y=\zeta-\zeta_{d}+\varepsilon(\cos \theta-1) \\
\dot{y}=\dot{\zeta}-\varepsilon \dot{\theta} \sin \theta
\end{gathered}
$$

(recall that in the stabilization context considered in this work, the horizontal and vertical desired positions $\xi_{d}$ and $\zeta_{d}$ are considered constant), $\arctan (a, b)$ represents the (unique) angle $\phi$ such that $\sin \phi=a / \sqrt{a^{2}+b^{2}}$ and $\cos \phi=b / \sqrt{a^{2}+b^{2}} ; k_{i j}$, $i=1,2,3, j=1,2$, are positive constants; $k_{0}$ is a positive constant less than unity, i.e.

$$
0<k_{0}<1
$$

$\sigma_{0}$ is a linear saturation for $\left(L_{0}, M_{0}\right)$, and $\sigma_{11}, \sigma_{12}, \sigma_{21}$, and $\sigma_{22}$ are strictly increasing twice continuously differentiable generalized saturations with bounds $M_{11}, M_{12}, M_{21}$, and limit bounds $M_{22}^{+}$and $M_{22}^{-}$such that

$$
\begin{gathered}
\sqrt{M_{12}^{2}+\left(1+M_{22}^{-}\right)^{2}}+\varepsilon M_{0}^{2} \leq U_{1} \\
M_{22}^{+}<1
\end{gathered}
$$

$\sigma_{30}$ is a linear saturation for $\left(L_{30}, M_{30}\right), \sigma_{31}$ a homogeneous saturation for $\left(\alpha_{31}, r_{31}, M_{31}\right)$, and, for every $j \in\{2,3\}, \sigma_{3 j}$ is a strictly increasing strictly passive function being locally Lipschitz on $\mathbb{R} \backslash\{0\}$ and locally $r_{j}$-homogeneous of degree $\alpha_{j}$ satisfying

$$
B_{33} \triangleq \lim _{s \rightarrow \infty} \max \left\{\sigma_{33}\left(M_{31}^{+}+\sigma_{32}(s)\right),-\sigma_{33}\left(-M_{31}^{-}+\sigma_{32}(-s)\right)\right\}<\infty
$$

—notice that the satisfaction of (22a) implies that among $\sigma_{32}$ and $\sigma_{33}$, at least one of them must be bounded (and, consequently, a strictly increasing homogeneous saturation)—, such that ${ }^{2}$

$$
\begin{gathered}
M_{30}+B_{33} \leq U_{2} \\
\lim _{s \rightarrow-\infty} \sigma_{33}(s)<-M_{30}^{-}, \quad \lim _{s \rightarrow \infty} \sigma_{33}(s)>M_{30}^{+} \\
\lim _{s \rightarrow-\infty} \sigma_{32}(s)<-M_{31}^{-}+\sigma_{33}^{-1}\left(-M_{30}^{-}\right) \quad, \quad \lim _{s \rightarrow \infty} \sigma_{32}(s)>M_{31}^{+}+\sigma_{33}^{-1}\left(M_{30}^{+}\right) \\
r_{33}=\alpha_{31}=\alpha_{32} \quad, \quad \alpha_{33}=2 r_{32}-r_{31}>0>r_{32}-r_{31}
\end{gathered}
$$

and $\dot{\theta}_{d}$ and $\ddot{\theta}_{d}$ are given by

$$
\dot{\theta}_{d}=k_{0} \dot{\bar{\theta}}_{d}
$$

\footnotetext{
${ }^{2}$ Let us note that their strictly increasing character renders $\sigma_{32}$ and $\sigma_{33}$ invertible functions mapping $\mathbb{R}$ onto $\sigma_{32}(\mathbb{R})$ and $\sigma_{33}(\mathbb{R})$ respectively, and consequently $\sigma_{32}^{-1}$ and $\sigma_{33}^{-1}$ are well-defined functions respectively mapping $\sigma_{32}(\mathbb{R})$ and $\sigma_{33}(\mathbb{R})$ onto $\mathbb{R}$. In particular, observe that, by $(22 \mathrm{c})$, we have that $M_{30}^{+} \in \sigma_{33}(\mathbb{R})$ and $-M_{30}^{-} \in \sigma_{33}(\mathbb{R})$.
} 
with

$$
\dot{\bar{\theta}}_{d}=\frac{\bar{v}_{1} \dot{v}_{2}-\left(1+v_{2}\right) \dot{\bar{v}}_{1}}{\bar{u}_{1}^{2}}
$$

and

$$
\ddot{\theta}_{d}=k_{0} \ddot{\bar{\theta}}_{d}
$$

with

$$
\ddot{\bar{\theta}}_{d}=\frac{\bar{v}_{1} \ddot{v}_{2}-\left(1+v_{2}\right) \ddot{\bar{v}}_{1}}{\bar{u}_{1}^{2}}-\frac{2 \dot{\bar{u}}_{1} \dot{\bar{\theta}}_{d}}{\bar{u}_{1}}
$$

where $\bar{v}_{1} \triangleq v_{1} / k_{0}, \dot{\bar{v}}_{1}, \ddot{\bar{v}}_{1}, \dot{v}_{2}, \ddot{v}_{2}$, and $\dot{\bar{u}}_{1}$ are given by

$$
\begin{gathered}
\bar{v}_{1}=-\sigma_{12}\left(s_{12}\right) \\
\dot{\bar{v}}_{1}=-\sigma_{12}^{\prime}\left(s_{12}\right) \dot{s}_{12} \\
\ddot{\bar{v}}_{1}=-\sigma_{12}^{\prime \prime}\left(s_{12}\right) \dot{s}_{12}^{2}-\sigma_{12}^{\prime}\left(s_{12}\right) \ddot{s}_{12} \\
\dot{v}_{2}=-\sigma_{22}^{\prime}\left(s_{22}\right) \dot{s}_{22} \\
\ddot{v}_{2}=-\sigma_{22}^{\prime \prime}\left(s_{22}\right) \dot{s}_{22}^{2}-\sigma_{22}^{\prime}\left(s_{22}\right) \ddot{s}_{22} \\
\dot{\bar{u}}_{1}=\frac{v_{1} \dot{v}_{1}+\left(1+v_{2}\right) \dot{v}_{2}}{\bar{u}_{1}}
\end{gathered}
$$

with $\dot{v}_{1} \triangleq k_{0} \dot{\bar{v}}_{1}$, and $s_{i 2}, \dot{s}_{i 2}$, and $\ddot{s}_{i 2}, i=1,2$, given by

$$
\begin{gathered}
s_{12}=k_{12} \dot{x}+\sigma_{11}\left(k_{11} x\right) \\
\dot{s}_{12}=k_{12} a_{x}+\sigma_{11}^{\prime}\left(k_{11} x\right) k_{11} \dot{x} \\
\ddot{s}_{12}=k_{12} \dot{a}_{x}+\sigma_{11}^{\prime \prime}\left(k_{11} x\right)\left(k_{11} \dot{x}\right)^{2}+\sigma_{11}^{\prime}\left(k_{11} x\right) k_{11} a_{x} \\
s_{22}=k_{22} \dot{y}+\sigma_{21}\left(k_{21} y\right) \\
\dot{s}_{22}=k_{22} a_{y}+\sigma_{21}^{\prime}\left(k_{21} y\right) k_{21} \dot{y} \\
\ddot{s}_{22}=k_{22} \dot{a}_{y}+\sigma_{21}^{\prime \prime}\left(k_{21} y\right)\left(k_{21} \dot{y}\right)^{2}+\sigma_{21}^{\prime}\left(k_{21} y\right) k_{21} a_{y}
\end{gathered}
$$

and $a_{x}, \dot{a}_{x}, a_{y}$, and $\dot{a}_{y}$ given $\mathrm{by}^{3}$

$$
\begin{gathered}
a_{x}=-\bar{u}_{1} \sin \theta \\
\dot{a}_{x}=-\dot{\bar{u}}_{1} \sin \theta-\bar{u}_{1} \dot{\theta} \cos \theta \\
a_{y}=\bar{u}_{1} \cos \theta-1 \\
\dot{a}_{y}=\dot{\bar{u}}_{1} \cos \theta-\bar{u}_{1} \dot{\theta} \sin \theta
\end{gathered}
$$

Let us note that the design of the proposed scheme is based on the observation that the (actual, transformed and auxiliary) input expressions in Eqs. (14)-(17) define $\theta=\theta_{d}(x, \dot{x}, y, \dot{y})$ (with $\theta_{d}$ as in Eq. (19)) as a manifold where the translational motion dynamics has a globally asymptotically stable equilibrium at the desired configuration, with the consequent trajectories simultaneously leading the rotational motion variables to zero. Hence, through the control law in Eq. (18) (under the consideration of the rest of the expressions and conditions involved in the algorithm), $\theta$ is made attain $\theta_{d}$ after a finite time avoiding discontinuities throughout the scheme (contrarily, for instance, to sliding-mode-based approaches). The control objective is thus achieved. Moreover, through the control expressions in Eqs. (14)-(15), $u_{1}$ is ensured to remain positive in accordance to the unidirectional nature of the thrust. Furthermore, through the use of suitably bounded functions $\sigma_{i j}$ in the definition of the auxiliary and torque inputs in Eqs. (16)-(18), input saturation is avoided. Such analytical features of the proposed scheme are formally stated a proven next.

Proposition 1. Consider the PVTOL aircraft dynamics in Eqs. (1) with input saturation bounds $U_{1}>1$ and $U_{2}>0$. Let the input thrust $u_{1}$ be defined as in (14), with the involved auxiliary (input) variables $\bar{u}_{1}, v_{1}$, and $v_{2}$ in turn defined as in Eqs. (15)-(17), under the satisfaction of inequalities (21), and positive gains $k_{i j}, i, j=1,2$. Let the input rolling moment $u_{2}$ be defined as in (18), where $\theta_{d}, \dot{\theta}_{d}$, and $\ddot{\theta}_{d}$ are defined through Eqs. (19), (23)-(24), with the involved intermediate (auxiliary)

\footnotetext{
${ }^{3}$ Let us note that the dotted $\left({ }^{*}\right)$ and double-dotted $\left({ }^{*}\right)$ variables defined through Eqs. (23)-(27) do not a priori correspond to the first- and second-order change rate of the referred variables. However, such a correspondence will be later on proven to (ultimately) hold from some finite time $t_{1}$ on.
} 
variables in turn defined as in Eqs. (25)-(27), under the fulfillment of conditions (22), and positive gains $k_{3 l}, l=1,2$. Then, for any $(\xi, \zeta, \theta, \dot{\xi}, \dot{\zeta}, \dot{\theta})(0) \in \mathbb{R}^{6}$ :

1. $0<1-M_{22}^{+} \leq u_{1}(t)<B_{u_{1}} \triangleq \sqrt{\left(k_{0} M_{12}\right)^{2}+\left(1+M_{22}^{-}\right)^{2}}+\varepsilon M_{0}^{2}<U_{1}$ and $\left|u_{2}(t)\right|<B_{u_{2}} \triangleq M_{30}+B_{33} \leq U_{2}, \forall t \geq 0$;

2. the closed-loop system solutions $(\xi, \zeta, \theta)(t)$ are bounded on $[0, \tau]$ for any $\tau \in(0, \infty)$;

3. there exist initial-condition-independent positive constants $B_{\dot{\bar{v}}_{1}}, B_{\dot{v}_{2}}, B_{\dot{\bar{u}}_{1}}$, and $B_{\dot{\bar{\theta}}_{d}}$ such that (along the closed-loop system trajectories) $\left|\dot{v}_{1}(t)\right|<\left|\dot{\bar{v}}_{1}(t)\right| \leq B_{\dot{\bar{v}}_{1}},\left|\dot{v}_{2}(t)\right| \leq B_{\dot{v}_{2}},\left|\dot{\bar{u}}_{1}(t)\right| \leq B_{\dot{\bar{u}}_{1}}$, and $\left|\dot{\theta}_{d}(t)\right|<\left|\overline{\bar{\theta}}_{d}(t)\right| \leq B_{\dot{\bar{\theta}}_{d}}, \forall t \geq 0$;

4. there exists a finite time $t_{1} \geq 0$ such that:

a) $|\dot{\theta}(t)| \leq B_{\dot{\theta}}, \forall t \geq t_{1}$,

b) $\left|\ddot{\bar{v}}_{1}(t)\right| \leq B_{\ddot{\bar{v}}_{1}},\left|\ddot{v}_{2}(t)\right| \leq B_{\ddot{v}_{2}},\left|\ddot{\theta}_{d}(t)\right| \leq k_{0} B_{\ddot{\bar{\theta}}_{d}}, \forall t \geq t_{1}$,

for some initial-condition-independent positive constants $B_{\dot{\theta}}, B_{\vec{v}_{1}}, B_{\ddot{v}_{2}}$, and $B_{\dot{\theta}_{d}}$;

5. provided that $k_{0}$ and $k_{i j}, i, j=1,2$, are sufficiently small and $k_{32}$ is sufficiently high, from $t_{1}$ on, $\theta_{d}(t)$ is globally finite-time stabilized in the rotational coordinate space, i.e. $\theta_{d}(t)$ becomes a stable solution of the rotational motion closed-loop dynamics and, for any $(\theta, \dot{\theta})\left(t_{1}\right) \in \mathbb{R}^{2}$, there exists a finite time $t_{2} \geq t_{1}$ such that $\theta(t)=\theta_{d}(t), \forall t \geq t_{2}$;

6. provided that $\varepsilon$ is sufficiently small, from $t_{2}$ on, $(\xi, \zeta)(t) \equiv(0,0)$ becomes a stable solution of the translational motion closed-loop dynamics and, for any $(\xi, \zeta, \dot{\xi}, \dot{\zeta})\left(t_{2}\right) \in \mathbb{R}^{4},(\xi, \zeta, \theta)(t) \rightarrow(0,0,0)$ as $t \rightarrow \infty$.

Proof:

1. Item 1 of the statement follows directly from the definition of $u_{1}, u_{2}, \bar{u}_{1}, v_{1}$, and $v_{2}$ in Eqs. (14)-(18), the consideration of inequalities (21) and (22b), and the strictly increasing character of $\sigma_{i j}, i, j=1,2, \sigma_{32}$ and $\sigma_{33}$. Its proof is consequently straightforward.

2. Observe from the system dynamics in Eqs. (4), the control expression defined for $\bar{u}_{1}$ (Eq. (15)), and inequality (21a) that

$$
\begin{aligned}
&|\ddot{x}(t)|<\sqrt{M_{12}^{2}+\left(1+M_{22}^{-}\right)^{2}} \triangleq B_{\bar{u}_{1}} \\
&|\ddot{y}(t)|<B_{\bar{u}_{1}}+1 \\
&|\ddot{\theta}(t)|<M_{30}+B_{33}=B_{u_{2}}
\end{aligned}
$$

Hence, for any $\tau \in(0, \infty)$ :

$$
\begin{aligned}
|\dot{x}(t)|<|\dot{x}(0)|+B_{\bar{u}_{1}} \tau & , & |x(t)|<|x(0)|+|\dot{x}(0)| \tau+B_{\bar{u}_{1}} \tau^{2} / 2 \\
|\dot{y}(t)|<|\dot{y}(0)|+\left(B_{\bar{u}_{1}}+1\right) \tau, & & |y(t)|<|y(0)|+|\dot{y}(0)| \tau+\left(B_{\bar{u}_{1}}+1\right) \tau^{2} / 2 \\
|\dot{\theta}(t)|<|\dot{\theta}(0)|+B_{u_{2}} \tau \quad & , & |\theta(t)|<|\theta(0)|+|\dot{\theta}(0)| \tau+B_{u_{2}} \tau^{2} / 2
\end{aligned}
$$

$\forall t \in[0, \tau]$. Thus, from equations (2), we get that for any $\tau \in(0, \infty)$ :

$$
\begin{aligned}
|\xi(t)|<|\xi(0)|+2 \varepsilon+(|\dot{\xi}(0)|+\varepsilon|\dot{\theta}(0)|) \tau+B_{\bar{u}_{1}} \tau^{2} / 2 \\
|\zeta(t)|<|\zeta(0)|+4 \varepsilon+(|\dot{\zeta}(0)|+\varepsilon|\dot{\theta}(0)|) \tau+\left(B_{\bar{u}_{1}}+1\right) \tau^{2} / 2 \\
|\theta(t)|<|\theta(0)|+|\dot{\theta}(0)| \tau+B_{u_{2}} \tau^{2} / 2
\end{aligned}
$$

3. Note that Eq. (25b) may be rewritten as

$$
\dot{\bar{v}}_{1}=-\sigma_{12}^{\prime}\left(s_{12}\right)\left[k_{12} a_{x}+\frac{k_{11}}{k_{12}} \sigma_{11}^{\prime}\left(k_{11} x\right)\left(s_{12}-\sigma_{11}\left(k_{11} x\right)\right)\right]
$$

Then, by applying Lemma 1, we have that (along the closed-loop system trajectories)

$$
\left|\dot{\bar{v}}_{1}(t)\right| \leq k_{12} A_{12}^{0,1} B_{\bar{u}_{1}}+\frac{k_{11}}{k_{12}} A_{11}^{0,1}\left(A_{12}^{1,1}+A_{12}^{0,1} M_{11}\right) \triangleq B_{\dot{\bar{v}}_{1}}
$$

$\forall t \geq 0$; recall further that $v_{1}=k_{0} \bar{v}_{1}$ and consequently, under the consideration of (21a), we have that

$$
\left|\dot{v}_{1}(t)\right|<\left|\dot{\bar{v}}_{1}(t)\right| \leq B_{\dot{\bar{v}}_{1}}
$$

$\forall t \geq 0$. Following an analog procedure for Eq. (25d), we get

$$
\left|\dot{v}_{2}(t)\right| \leq k_{22} A_{22}^{0,1}\left(B_{\bar{u}_{1}}+1\right)+\frac{k_{21}}{k_{22}} A_{21}^{0,1}\left(A_{22}^{1,1}+A_{22}^{0,1} M_{21}\right) \triangleq B_{\dot{v}_{2}}
$$

$\forall t \geq 0$. Observe now that Eq. (25f) may be rewritten as

$$
\dot{\bar{u}}_{1}=\dot{v}_{2} \cos \theta_{d}-\dot{v}_{1} \sin \theta_{d}=\sqrt{\dot{v}_{1}^{2}+\dot{v}_{2}^{2}} \cos \left(\theta_{d}+\arctan \left(\dot{v}_{1}, \dot{v}_{2}\right)\right)
$$


wherefrom we have that

$$
\left|\dot{\bar{u}}_{1}(t)\right| \leq \sqrt{B_{\dot{\bar{v}}_{1}}^{2}+B_{\dot{v}_{2}}^{2}} \triangleq B_{\dot{\bar{u}}_{1}}
$$

$\forall t \geq 0$. Furthermore, notice that Eq. (23b) may be rewritten as

$$
\dot{\bar{\theta}}_{d}=\frac{\bar{v}_{1} \dot{v}_{2}}{\bar{u}_{1}^{2}}-\frac{\dot{\bar{v}}_{1} \cos \theta_{d}}{\bar{u}_{1}}
$$

whence we get that

$$
\left|\dot{\bar{\theta}}_{d}(t)\right| \leq \frac{M_{12} B_{\dot{v}_{2}}}{\left(1-M_{22}^{+}\right)^{2}}+\frac{B_{\dot{\bar{v}}_{1}}}{1-M_{22}^{+}} \triangleq B_{\dot{\bar{\theta}}_{d}}
$$

$\forall t \geq 0$. Observe further from Eq. (23a) that, under the consideration of (21a), we have that

$$
\left|\dot{\theta}_{d}(t)\right|<\left|\dot{\bar{\theta}}_{d}(t)\right| \leq B_{\dot{\bar{\theta}}_{d}}
$$

$\forall t \geq 0$.

4a. Let $\sigma_{34}$ be a generalized saturation function with limit bounds such that ${ }^{4}$

$$
M_{34}^{-}<-\lim _{s \rightarrow-\infty} \sigma_{33}\left(M_{31}^{-}+\sigma_{32}(s)\right)-M_{30}^{-}
$$

and

$$
M_{34}^{+}<\lim _{s \rightarrow \infty} \sigma_{33}\left(-M_{31}^{+}+\sigma_{32}(s)\right)-M_{30}^{+}
$$

Let us further define the positive scalar function $V_{1}=\dot{\theta}^{2} / 2$. Its derivative along the trajectories of the closed-loop rotational motion dynamics is given by

$$
\dot{V}_{1}=\dot{\theta} \ddot{\theta}=\dot{\theta}\left[\sigma_{30}\left(\ddot{\theta}_{d}\right)-\sigma_{33}\left(\sigma_{31}\left(k_{31}\left(\theta-\theta_{d}\right)\right)+\sigma_{32}\left(k_{32}\left(\dot{\theta}-\dot{\theta}_{d}\right)\right)\right)\right]
$$

which may be rewritten as

$$
\dot{V}_{1}=-\dot{\theta} \sigma_{34}(\dot{\theta})+\dot{\theta}\left[\sigma_{30}\left(\ddot{\theta}_{d}\right)-\sigma_{33}\left(\sigma_{31}\left(k_{31}\left(\theta-\theta_{d}\right)\right)+\sigma_{32}\left(k_{32}\left(\dot{\theta}-\dot{\theta}_{d}\right)\right)\right)+\sigma_{34}(\dot{\theta})\right]
$$

Observe, from item 3 of the statement, inequalities (32), and the strictly increasing character of $\sigma_{32}$ and $\sigma_{33},{ }^{5}$ that

$$
\begin{gathered}
\dot{\theta} \geq B_{\dot{\theta}}^{+} \triangleq \frac{\sigma_{32}^{-1}\left(M_{31}^{+}+\sigma_{33}^{-1}\left(M_{30}^{+}+M_{34}^{+}\right)\right)}{k_{32}}+B_{\dot{\bar{\theta}}_{d}}>0 \\
\Longrightarrow \dot{\theta}-\dot{\theta}_{d} \geq \frac{\sigma_{32}^{-1}\left(M_{31}^{+}+\sigma_{33}^{-1}\left(M_{30}^{+}+M_{34}^{+}\right)\right)}{k_{32}}+B_{\dot{\bar{\theta}}_{d}}-\dot{\theta}_{d} \\
\quad \geq \frac{\sigma_{32}^{-1}\left(M_{31}^{+}+\sigma_{33}^{-1}\left(M_{30}^{+}+M_{34}^{+}\right)\right)}{k_{32}} \\
\Longrightarrow \sigma_{32}\left(k_{32}\left(\dot{\theta}-\dot{\theta}_{d}\right)\right) \geq M_{31}^{+}+\sigma_{33}^{-1}\left(M_{30}^{+}+M_{34}^{+}\right) \\
\Longrightarrow \sigma_{32}(\cdot)+\sigma_{31}(\cdot) \geq \sigma_{33}^{-1}\left(M_{30}^{+}+M_{34}^{+}\right)+M_{31}^{+}+\sigma_{31}(\cdot) \\
\Longrightarrow \sigma_{33}^{-1}\left(M_{30}^{+}+M_{34}^{+}\right) \\
\Longrightarrow \sigma_{33}\left(\sigma_{32}(\cdot)+\sigma_{31}(\cdot)\right) \geq M_{30}^{+}+M_{34}^{+} \\
\left.\Longrightarrow \sigma_{32}(\cdot)+\sigma_{31}(\cdot)\right)-\sigma_{30}(\cdot)-\sigma_{34}(\cdot) \geq M_{30}^{+}-\sigma_{30}(\cdot)+M_{34}^{+}-\sigma_{34}(\cdot) \geq 0 \\
\Longrightarrow \sigma_{33}\left(\sigma_{31}\left(k_{31}\left(\theta-\theta_{d}\right)\right)+\sigma_{32}\left(k_{32}\left(\dot{\theta}-\dot{\theta}_{d}\right)\right)\right)+\sigma_{34}(\dot{\theta}) \leq 0
\end{gathered}
$$

while analogous developments show that

$$
\begin{aligned}
\dot{\theta} \leq B_{\dot{\theta}}^{-} \triangleq & \frac{\sigma_{32}^{-1}\left(-M_{31}^{-}+\sigma_{33}^{-1}\left(-M_{30}^{-}-M_{34}^{-}\right)\right)}{k_{32}}-B_{\dot{\bar{\theta}}_{d}}<0 \\
& \Longrightarrow \sigma_{30}\left(\ddot{\theta}_{d}\right)-\sigma_{33}\left(\sigma_{31}\left(k_{31}\left(\theta-\theta_{d}\right)\right)+\sigma_{32}\left(k_{32}\left(\dot{\theta}-\dot{\theta}_{d}\right)\right)\right)+\sigma_{34}(\dot{\theta}) \geq 0
\end{aligned}
$$

\footnotetext{
${ }^{4}$ Let us note that the satisfaction of inequalities (22c) and (22d) ensures positivity of the right-hand-side expressions of inequalities (32).

${ }^{5}$ Recall that, in view of the strictly increasing character of $\sigma_{32}$ and $\sigma_{33}, \sigma_{32}^{-1}$ and $\sigma_{33}^{-1}$ are well-defined functions respectively mapping $\sigma_{32}(\mathbb{R})$ and $\sigma_{33}(\mathbb{R})$ onto $\mathbb{R}$. In particular, by inequalities (32), we have that $M_{30}^{+}+M_{34}^{+} \in \sigma_{33}(\mathbb{R}),-M_{30}^{-}-M_{34}^{-} \in \sigma_{33}(\mathbb{R}), M_{31}^{+}+\sigma_{33}^{-1}\left(M_{30}^{+}+M_{34}^{+}\right) \in \sigma_{32}(\mathbb{R})$, and $-M_{31}^{-}+\sigma_{33}^{-1}\left(-M_{30}^{-}-\right.$ $\left.M_{34}^{-}\right) \in \sigma_{32}(\mathbb{R})$.
} 
From these expressions we see that

$$
|\dot{\theta}| \geq \max \left\{B_{\dot{\theta}}^{+},-B_{\dot{\theta}}^{-}\right\}
$$

$$
\Longrightarrow \dot{\theta}\left[\sigma_{30}\left(\ddot{\theta}_{d}\right)-\sigma_{33}\left(\sigma_{31}\left(k_{31}\left(\theta-\theta_{d}\right)\right)+\sigma_{32}\left(k_{32}\left(\dot{\theta}-\dot{\theta}_{d}\right)\right)\right)+\sigma_{34}(\dot{\theta})\right] \leq 0
$$

whence, in view of (33), we conclude that

$$
\dot{V}_{1} \leq-\dot{\theta} \sigma_{34}(\dot{\theta}) \quad \forall|\dot{\theta}| \geq \max \left\{B_{\dot{\theta}}^{+},-B_{\dot{\theta}}^{-}\right\}
$$

with $\dot{\theta} \sigma_{34}(\dot{\theta})$ being a positive definite function of $\dot{\theta}$ in view of the strictly passive character of $\sigma_{34}$. Then, according to [Khalil (2002), Theorem 4.18], ${ }^{6}$ there exists a finite time $t_{1} \geq 0$ such that

$$
|\dot{\theta}(t)| \leq \max \left\{B_{\dot{\theta}}^{+},-B_{\dot{\theta}}^{-}\right\} \triangleq B_{\dot{\theta}}
$$

$\forall t \geq t_{1}$.

4b. Notice that Eq. (25c) may be rewritten as

$$
\begin{aligned}
& \ddot{\bar{v}}_{1}=-\sigma_{12}^{\prime \prime}\left(s_{12}\right)[\left.-k_{12} \bar{u}_{1} \sin \theta+\frac{k_{11}}{k_{12}} \sigma_{11}^{\prime}\left(k_{11} x\right)\left(s_{12}-\sigma_{11}\left(k_{11} x\right)\right)\right]^{2} \\
&-\sigma_{12}^{\prime}\left(s_{12}\right)\left[-\left(k_{12} \dot{\bar{u}}_{1}+k_{11} \bar{u}_{1} \sigma_{11}^{\prime}\left(k_{11} x\right)\right) \sin \theta-k_{12} \bar{u}_{1} \dot{\theta} \cos \theta\right. \\
&\left.+\left(\frac{k_{11}}{k_{12}}\right)^{2} \sigma_{11}^{\prime \prime}\left(k_{11} x\right)\left(s_{12}-\sigma_{11}\left(k_{11} x\right)\right)^{2}\right]
\end{aligned}
$$

Thus, by applying Lemma 1 and considering items 3 and 4a of the statement, we obtain (along the closed-loop system trajectories)

$$
\begin{aligned}
\left|\ddot{\bar{v}}_{1}(t)\right| \leq k_{12}^{2} A_{12}^{0,2} B_{\bar{u}_{1}}^{2}+2 k_{11} B_{\bar{u}_{1}} A_{11}^{0,1}\left(A_{12}^{1,2}+A_{12}^{0,2} M_{11}\right) & \\
& +\left(\frac{k_{11} A_{11}^{0,1}}{k_{12}}\right)^{2}\left(A_{12}^{2,2}+2 A_{12}^{1,2} M_{11}+A_{12}^{0,2} M_{11}^{2}\right)+A_{12}^{0,1} C_{1} \\
& +\left(\frac{k_{11}}{k_{12}}\right)^{2} A_{11}^{0,2}\left(A_{12}^{2,1}+2 A_{12}^{1,1} M_{11}+A_{12}^{0,1} M_{11}^{2}\right) \triangleq B_{\overline{\vec{v}}_{1}}
\end{aligned}
$$

$\forall t \geq t_{1}$, with

$$
C_{1} \triangleq \sqrt{\left(k_{12} B_{\overline{\bar{u}}_{1}}+k_{11} B_{\bar{u}_{1}} A_{11}^{0,1}\right)^{2}+\left(k_{12} B_{\bar{u}_{1}} B_{\dot{\theta}}\right)^{2}}
$$

Following a similar procedure for Eq. (25e), we get

$$
\begin{aligned}
\left|\ddot{v}_{2}(t)\right| \leq k_{22}^{2} A_{22}^{0,2}\left(B_{\bar{u}_{1}}+1\right)^{2} & +2 k_{21}\left(B_{\bar{u}_{1}}+1\right) A_{21}^{0,1}\left(A_{22}^{1,2}+A_{22}^{0,2} M_{21}\right) \\
& +\left(\frac{k_{21} A_{21}^{0,1}}{k_{22}}\right)^{2}\left(A_{22}^{2,2}+2 A_{22}^{1,2} M_{21}+A_{22}^{0,2} M_{21}^{2}\right)+A_{22}^{0,1}\left(C_{2}+k_{21} A_{21}^{0,1}\right) \\
& +\left(\frac{k_{21}}{k_{22}}\right)^{2} A_{21}^{0,2}\left(A_{22}^{2,1}+2 A_{22}^{1,1} M_{21}+A_{22}^{0,1} M_{21}^{2}\right) \triangleq B_{\ddot{v_{2}}}
\end{aligned}
$$

$\forall t \geq t_{1}$, with

$$
C_{2} \triangleq \sqrt{\left(k_{22} B_{\dot{\bar{u}}_{1}}+k_{21} B_{\bar{u}_{1}} A_{21}^{0,1}\right)^{2}+\left(k_{22} B_{\bar{u}_{1}} B_{\dot{\theta}}\right)^{2}}
$$

Furthermore, note that Eq. (24b) may be rewritten as

$$
\ddot{\bar{\theta}}_{d}=\frac{\bar{v}_{1} \ddot{v}_{2}}{\bar{u}_{1}^{2}}-\frac{\ddot{\bar{v}}_{1} \cos \theta_{d}+2 \dot{\bar{u}}_{1} \dot{\bar{\theta}}_{d}}{\bar{u}_{1}}
$$

whence we get that

$$
\left|\ddot{\bar{\theta}}_{d}(t)\right| \leq \frac{M_{12} B_{\ddot{v}_{2}}}{\left(1-M_{22}^{+}\right)^{2}}+\frac{B_{\ddot{\bar{v}}_{1}}+2 B_{\dot{\bar{u}}_{1}} B_{\dot{\bar{\theta}}_{d}}}{1-M_{22}^{+}} \triangleq B_{\ddot{\bar{\theta}}_{d}}
$$

\footnotetext{
${ }^{6}$ Theorem 4.18 of [Khalil (2002)] is being applied by considering the closed-loop rotational motion dynamics a first order subsystem with respect to $\dot{\theta}$, i.e. $\frac{d}{d t} \dot{\theta}=u_{2}(t, \dot{\theta})$ where (along the closed loop trajectories) the rest of the system variables, involved in $u_{2}$, are considered time-varying functions.
} 
$\forall t \geq t_{1}$. Hence, from Eq. (24a), we conclude that

$$
\left|\ddot{\theta}_{d}(t)\right|=k_{0}\left|\ddot{\bar{\theta}}_{d}(t)\right| \leq k_{0} B_{\ddot{\theta}_{d}}
$$

$\forall t \geq t_{1}$.

5. Note, from the proof of item $4 \mathrm{a}$ (more precisely, see expressions (31) and (34)), that with sufficiently small gains $k_{i j}$, $i, j=1,2$, and a high enough value of $k_{32}$ - such that $^{7} B_{\dot{\theta}} \leq L_{0}$ - we have (along the system trajectories) that $\sigma_{0}(\dot{\theta}(t))=$ $\dot{\theta}(t), \forall t \geq t_{1}$. Hence, from $t_{1}$ on, $u_{1}$ in (14) adopts the form expressed in (3) and consequently the system dynamics in Eqs. (4) becomes an equivalent representation of the system model in Eqs. (1) under the coordinate and input variable changes defined through Eqs. (2) and (3). This in turn entails that, from $t_{1}$ on, the dotted ( ') and double-dotted ( $\left.{ }^{*}\right)$ variables defined through Eqs. (23)-(27) correspond to the first- and second-order change rate of the referred variables; in particular $\dot{\theta}_{d}(t)=\frac{d}{d t} \theta_{d}(t)$ and $\ddot{\theta}_{d}(t)=\frac{d}{d t} \dot{\theta}_{d}(t), \forall t \geq t_{1}$. Furthermore, from items $4 \mathrm{~b}$ of the statement and 4 of Definition 4 , one sees that by choosing a sufficiently small value of $k_{0}$ - such that $k_{0} B_{\ddot{\theta}_{d}} \leq L_{30}$-, we obtain (along the system trajectories) $\sigma_{30}\left(\ddot{\theta}_{d}(t)\right)=\ddot{\theta}_{d}(t), \forall t \geq t_{1}$. Then, from $t_{1}$ on, the rotational motion dynamics becomes

$$
\ddot{\theta}=\ddot{\theta}_{d}-\sigma_{33}\left(\sigma_{31}\left(k_{31}\left(\theta-\theta_{d}\right)\right)+\sigma_{32}\left(k_{32}\left(\dot{\theta}-\dot{\theta}_{d}\right)\right)\right)
$$

By defining $e_{1}=\theta-\theta_{d}$ and $e_{2}=\dot{\theta}-\dot{\theta}_{d}$, this subsystem adopts a state-space representation of the form

$$
\begin{aligned}
& \dot{e}_{1}=e_{2} \\
& \dot{e}_{2}=-\sigma_{33}\left(\sigma_{31}\left(k_{31} e_{1}\right)+\sigma_{32}\left(k_{32} e_{2}\right)\right)
\end{aligned}
$$

Thus, under the satisfaction of (22e), by Lemma 4 and Remark 2 , we conclude that $\left(e_{1}, e_{2}\right)=(0,0)$ is a globally finite-time stable equilibrium of this subsystem. Hence, $\theta_{d}(t)$ becomes a globally finite-time stable solution of the rotational motion closed-loop dynamics, or equivalently, it becomes a stable solution of the referred subsystem and, for any $(\theta, \dot{\theta})\left(t_{1}\right) \in \mathbb{R}^{2}$, there exists a finite time $t_{2} \geq t_{1}$ such that $\theta(t)=\theta_{d}(t), \forall t \geq t_{2}$.

6. Observe from item 2 of the statement that up to $t_{2}$ (and actually for any arbitrarily long finite time), the closed-loop system solutions exist and are bounded (which holds in both considered coordinate spaces). Further, from item 5 of the statement, the definitions of $\theta_{d}$ in (19) and $\bar{u}_{1}$ in (15), and Eqs. (4), one sees that, from $t_{2}$ on, we have that $\ddot{x}_{1}=-\bar{u}_{1} \sin \theta_{d}=v_{1}$ and $\ddot{y}_{1}=\bar{u}_{1} \cos \theta_{d}-1=v_{2}$ with $v_{1}$ and $v_{2}$ as defined in Eqs. (16)-(17), i.e. the translational motion closed-loop dynamics in the transformed coordinates becomes

$$
\begin{aligned}
& \ddot{x}=-k_{0} \sigma_{12}\left(k_{12} \dot{x}+\sigma_{11}\left(k_{11} x\right)\right) \\
& \ddot{y}=-\sigma_{22}\left(k_{22} \dot{y}+\sigma_{21}\left(k_{21} y\right)\right)
\end{aligned}
$$

By defining $z \triangleq(x, \dot{x}, y, \dot{y})^{T}$, this subsystem adopts a consequent state-space representation $\dot{z}=f(z)$ with $f\left(0_{4}\right)=0_{4}$. More precisely,

$$
\begin{aligned}
& \dot{z}_{1}=z_{2} \\
& \dot{z}_{2}=-k_{0} \sigma_{12}\left(k_{12} z_{2}+\sigma_{11}\left(k_{11} z_{1}\right)\right) \\
& \dot{z}_{3}=z_{4} \\
& \dot{z}_{4}=-\sigma_{22}\left(k_{22} z_{4}+\sigma_{21}\left(k_{21} z_{3}\right)\right)
\end{aligned}
$$

Let us now define the continuously differentiable scalar function

$$
V_{2}(z)=\frac{z_{2}^{2}}{2 k_{0}}+\int_{0}^{z_{1}} \sigma_{12}\left(\sigma_{11}\left(k_{11} s\right)\right) d s+\frac{z_{4}^{2}}{2}+\int_{0}^{z_{3}} \sigma_{22}\left(\sigma_{21}\left(k_{21} s\right)\right) d s
$$

Note, under the consideration of Lemma 2 and Remark 2, that $V_{2}(z)$ is radially unbounded and positive definite. Its derivative along the system trajectories is given by

$$
\begin{aligned}
\dot{V}_{2}(z)= & \frac{z_{2} \dot{z}_{2}}{k_{0}}+z_{2} \sigma_{12}\left(\sigma_{11}\left(k_{11} z_{1}\right)\right)+\frac{z_{4} \dot{z}_{4}}{k_{0}}+z_{4} \sigma_{22}\left(\sigma_{21}\left(k_{21} z_{3}\right)\right) \\
= & -z_{2}\left[\sigma_{12}\left(k_{12} z_{2}+\sigma_{11}\left(k_{11} z_{1}\right)\right)-\sigma_{12}\left(\sigma_{11}\left(k_{11} z_{1}\right)\right)\right] \\
& -z_{4}\left[\sigma_{22}\left(k_{22} z_{4}+\sigma_{21}\left(k_{21} z_{3}\right)\right)-\sigma_{22}\left(\sigma_{21}\left(k_{21} z_{3}\right)\right)\right]
\end{aligned}
$$

From Lemma 3 -in view of the strictly increasing character of $\sigma_{i 2}, i=1,2$ - one sees that $\dot{V}_{2}(z) \leq 0, \forall z \in \mathbb{R}^{4}$, with $\dot{V}_{2}(z)=0 \Longleftrightarrow z_{2}=z_{4}=0$, whence $0_{4}$ is concluded to be a stable equilibrium of the state equations (36), or equivalently

\footnotetext{
${ }^{7}$ Let us notice that generalized saturation functions with sufficiently low slope in $v_{1}$ and $v_{2}$ and small enough bounds in $u_{2}$ would also be helpful to get $B_{\dot{\theta}} \leq L_{0}$.
} 
$(x, y)(t) \equiv(0,0)$ is concluded to be a stable solution of subsystem (35). Further, from Eqs. (36) and the strictly passive character of the involved generalized saturation functions, one sees that $\left(z_{2}(t) \equiv 0\right) \wedge\left(z_{4}(t) \equiv 0\right) \Longrightarrow\left(\dot{z}_{2}(t) \equiv 0\right) \wedge\left(\dot{z}_{4}(t) \equiv\right.$ $0) \Longrightarrow\left(z_{1}(t) \equiv 0\right) \wedge\left(z_{3}(t) \equiv 0\right)$. Then, from La Salle's invariance principle, one concludes that, for any $z\left(t_{2}\right) \in \mathbb{R}^{4}$, $z(t) \rightarrow 0_{4}$ as $t \rightarrow \infty$. Moreover, observe from this asymptotic convergence that, since (along the closed-loop system trajectories $)(\theta, \dot{\theta})(t)=\left(\theta_{d}, \dot{\theta}_{d}\right)(t), \forall t \geq t_{2}$, and, as functions of the system variables, $\left.\theta_{d}(z)\right|_{z=0_{4}}=\left.\dot{\theta}_{d}(z)\right|_{z=0_{4}}=0$, then $(\theta, \dot{\theta})(t)=\left(\theta_{d}, \dot{\theta}_{d}\right)(t) \rightarrow 0_{2}$ as $t \rightarrow \infty$. Let us finally note that under the consideration of Eqs. (20) we have, from $t_{2}$ on, that $(\xi, \dot{\xi}, \zeta, \dot{\zeta})=T(z)$ with

$$
T(z)=\left(\begin{array}{c}
z_{1}+\varepsilon \sin \left(\theta_{d}(z)\right) \\
z_{2}+\varepsilon \dot{\theta}_{d}(z) \cos \left(\theta_{d}(z)\right) \\
z_{3}+\varepsilon\left[1-\cos \left(\theta_{d}(z)\right)\right] \\
z_{4}+\varepsilon \dot{\theta}_{d}(z) \cos \left(\theta_{d}(z)\right)
\end{array}\right)
$$

From this expression one gets that

$$
\left.\frac{\partial T}{\partial z}(z)\right|_{z=0_{4}}=\left(\begin{array}{cccc}
1+\varepsilon b_{1} & \varepsilon b_{2} & 0 & 0 \\
-\varepsilon b_{1} b_{2} & 1+\varepsilon\left(b_{1}-b_{2}^{2}\right) & 0 & 0 \\
0 & 0 & 1 & 0 \\
0 & 0 & 0 & 1
\end{array}\right)
$$

with $b_{1}=k_{0} k_{11} \sigma_{11}^{\prime}(0) \sigma_{12}^{\prime}(0)>0$ and $b_{2}=k_{0} k_{12} \sigma_{12}^{\prime}(0)>0$, and consequently $\left|\frac{\partial T}{\partial z}\left(0_{4}\right)\right|=\left(1+\varepsilon b_{1}\right)^{2}-\varepsilon b_{2}^{2}$ whence one can see that for sufficiently small $\varepsilon$ we have that $\left|\frac{\partial T}{\partial z}\left(0_{4}\right)\right| \neq 0$ and consequently $\frac{\partial T}{\partial z}\left(0_{4}\right)$ is non-singular. Hence, we conclude that, for a sufficiently small value of $\varepsilon$, stability holds for the trivial solution $(\xi, \zeta)(t) \equiv(0,0)$ in the original coordinates (see for instance [Khalil (2002), Exercise 4.26] and [Apostol (1974), Theorem 13.6]) and, from Remark 1, that for any $(\xi, \dot{\xi}, \zeta, \dot{\zeta})\left(t_{2}\right) \in \mathbb{R}^{4},(\xi, \zeta, \theta)(t) \rightarrow(0,0,0)$ as $t \rightarrow \infty$.

\section{OUTPUT FEEDBACK GLOBAL STABILIZER}

With $u \triangleq\left(u_{1}, u_{2}\right)^{T}$, let $u(\xi, \dot{\xi}, \zeta, \dot{\zeta}, \theta, \dot{\theta})$ represent the (state) feedback controller presented in the precedent section. Suppose now that position measurements are available while the velocity signals are not. In this case we show that the globally stabilizing objective is achievable through the precedent algorithm with the velocities replaced by estimation variables coming from a finitetime observer defined through a generalized dynamics that includes those used in [Frye et al.(2010)] and [Hong et al.(2001)] as particular cases. More specifically, we consider the closed loop generated by taking $u=u\left(\xi, \hat{w}_{2}, \zeta, \hat{w}_{4}, \theta, \hat{w}_{6}\right)$ under the additional consideration of the auxiliary dynamics

$$
\begin{aligned}
& \dot{\hat{w}}_{1}=\hat{w}_{2}+\sigma_{41}\left(k_{41}\left(\xi-\hat{w}_{1}\right)\right) \\
& \dot{\hat{w}}_{2}=-u_{1} \sin \theta+\varepsilon u_{2} \cos \theta+\sigma_{42}\left(k_{42}\left(\xi-\hat{w}_{1}\right)\right) \\
& \dot{\hat{w}}_{3}=\hat{w}_{4}+\sigma_{51}\left(k_{51}\left(\zeta-\hat{w}_{3}\right)\right) \\
& \dot{\hat{w}}_{4}=u_{1} \cos \theta+\varepsilon u_{2} \sin \theta-1+\sigma_{52}\left(k_{52}\left(\zeta-\hat{w}_{3}\right)\right) \\
& \dot{\hat{w}}_{5}=\hat{w}_{6}+\sigma_{61}\left(k_{61}\left(\theta-\hat{w}_{5}\right)\right) \\
& \dot{\hat{w}}_{6}=u_{2}+\sigma_{62}\left(k_{62}\left(\theta-\hat{w}_{5}\right)\right)
\end{aligned}
$$

where, for every $i \in\{4,5,6\}, k_{i 1}$ and $k_{i 2}$ are (arbitrary) positive constants, $\sigma_{i 1}(s)$ is a strictly passive function and $\sigma_{i 2}(s)$ is strongly passive, both being locally Lipschitz-continuous on $\mathbb{R} \backslash\{0\}$ and locally $r_{i}$-homogeneous of degree $\alpha_{i 1}$ and $\alpha_{i 2}$, respectively, for some $r_{i}$ such that

$$
\alpha_{i 2}=2 \alpha_{i 1}-r_{i}>0>\alpha_{i 1}-r_{i}
$$

The states of such an auxiliary dynamics will be proven to attain the variables of the PVTOL aircraft motion variables after a finite time. From that moment on, the conditions of the case treated in the precedent section are retrieved, consequently guaranteeing the control objective. Subsequently, we denote $w=(\xi, \dot{\xi}, \zeta, \dot{\zeta}, \theta, \dot{\theta})^{T}$, while we define $\hat{w} \triangleq\left(\hat{w}_{1}, \hat{w}_{2}, \hat{w}_{3}, \hat{w}_{4}, \hat{w}_{5}, \hat{w}_{6}\right)^{T}$.

Proposition 2. Assuming input saturation bounds $U_{1}>1$ and $U_{2}>0$, consider the PVTOL aircraft dynamics in Eqs. (1) with $u=u\left(\xi, \hat{w}_{2}, \zeta, \hat{w}_{4}, \theta, \hat{w}_{6}\right)$, i.e. in closed loop with the output-feedback scheme generated from the control algorithm considered in Proposition 1, with the horizontal, vertical, and rotational velocity variables in the control law expressions (14) and (18) respectively replaced by estimation variables $\hat{w}_{2}, \hat{w}_{4}$, and $\hat{w}_{6}$ dynamically computed through the auxiliary subsystem represented in Eqs. (37), under the satisfaction of the parametric conditions (38) (concerning the previously described functions $\sigma_{i 1}$ and $\left.\sigma_{i 2}, i=4,5,6\right)$ and the consideration of (arbitrary) positive constants $k_{i j}, i=4,5,6, j=1,2$. Then, for any $\left(w^{T}, \hat{w}^{T}\right)^{T}(0) \in \mathbb{R}^{12}$ : 
1. items 1 and 2 of Proposition 1 hold, i.e. the closed loop trajectories exist and are bounded at any finite time and, along them, input saturation is avoided;

2. there exists a finite time $t_{0} \geq 0$ such that $\hat{w}(t)=w(t), \forall t \geq t_{0}$;

3. from $t_{0}$ on, items 3-6 of Proposition 1 are retrieved with $t_{1} \geq t_{0}$, i.e. in particular, there exist a finite time $t_{1} \geq t_{0}$ such that $\left|\ddot{\theta}_{d}(t)\right| \leq k_{0} B_{\ddot{\theta}_{d}}, \forall t \geq t_{1}$, and a finite time $t_{2} \geq t_{1}$ such that, provided that $k_{0}$ and $k_{i j}, i, j=1,2$, are sufficiently small and $k_{32}$ is sufficiently high, $\theta(t)=\theta_{d}(t), \forall t \geq t_{2}$, and such that, provided that $\varepsilon$ is sufficiently small, from $t_{2}$ on, $(\xi, \zeta)(t) \equiv$ $(0,0)$ becomes a stable solution of the translational motion closed-loop dynamics and, for any $(\xi, \zeta, \dot{\xi}, \dot{\zeta})\left(t_{2}\right) \in \mathbb{R}^{4}$, $(\xi, \zeta, \theta)(t) \rightarrow(0,0,0)$ as $t \rightarrow \infty$.

Proof:

1. By reproducing the proof of items 1 and 2 of Proposition 1 under the consideration of estimation auxiliary states replacing the velocity variables in the control law expressions, one observes that both items hold, whence item 1 of the statement is concluded.

2. Let us define the observation error variables $\bar{w}_{i}=w_{i}-\hat{w}_{i}, i=1, \ldots, 6$. From the closed-loop system equations, the observation error variable dynamics is obtained as

$$
\begin{aligned}
& \dot{\bar{w}}_{i}=\bar{w}_{j}-\sigma_{i 1}\left(k_{i 1} \bar{w}_{i}\right) \\
& \dot{\bar{w}}_{j}=-\sigma_{j 2}\left(k_{j 2} \bar{w}_{i}\right)
\end{aligned}
$$

for all $i \in\{1,3,5\}$, with $j=i+1$. Hence, under the satisfaction of (38), by Lemma 5, item 2 of the statement is concluded.

3. Let us first note that in view of item 1 of the statement and the stability properties of the observation error dynamics, up to $t_{0}$ (and actually for any arbitrarily long finite time), all the closed-loop system variables, and consequently all the expressions involved in the definition of the control algorithm, exist and are bounded. On the other hand, in view of item 2 of the statement, from $t_{0}$ on, the state-feedback closed-loop dynamics considered in Proposition 1 is retrieved, and it is further mirrored by the auxiliary subsystem in Eqs. (37). Hence, from Proposition 1, item 3 of the statement is concluded.

\section{Simulation tests}

The proposed scheme was tested through simulation taking $\varepsilon=0.2$ and $\xi_{d}=\zeta_{d}=0$. Thrust and rolling moment saturation bounds $U_{1}=10$ and $U_{2}=10$ were considered (for the sake of simplicity, units will be omitted). Following the proposed design methodology, two controllers with different structures - more precisely, with different choices of $\sigma_{32}$ and $\sigma_{33}$ in $u_{2}$ - were implemented. The specific functions $\sigma_{0}(s), \sigma_{i j}(s), i, j=1,2, \sigma_{3 l}(s), l=0, \ldots, 3$, and $\sigma_{m n}(s), m=4,5,6, n=1,2$, that were involved at every implemented controller, are presented in Appendix $\mathrm{H}$. Their parameter values were fixed under the consideration of conditions (21) and (22) - for both controllers as: $M_{0}=0.7, M_{11}=M_{21}=3, M_{12}=7, M_{22}^{-}=6$, $M_{22}^{+}=0.9, M_{30}=M_{31}=2, \beta_{31}=1 / 3, \beta_{32}=1 / 2, \beta_{33}=1, \beta_{41}=\beta_{51}=\beta_{61}=2 / 3$, and $\beta_{42}=\beta_{52}=\beta_{62}=1 / 3$, while for controller 1: $M_{32}=6$ and $L_{32}=2$, and for controller $2: M_{33}=8$ and $L_{33}=3$. The control gains were taken as: $k_{0}=0.1$, $k_{11}=k_{21}=2, k_{12}=k_{22}=3$, and $k_{i j}=1, i=3, \ldots, 6, j=1,2$. For comparison purposes, the output-feedback algorithm of [Frye et al.(2010)] was implemented too with parameter values $\beta_{1}=\alpha_{2}=1 / 3, \beta_{2}=1 / 2, \alpha_{1}=2 / 3$, and control gains fixed as $k_{1}=k_{2}=1$; input saturation bounds were not included for this controller. The simulation was run taking initial conditions $w(0)=(\xi, \dot{\xi}, \zeta, \dot{\zeta}, \theta, \dot{\theta})(0)=(0,0,0,8,4 \pi, 0)$ and $\hat{w}(0)=(3,0,3,0,0,0)$. The results are shown in Fig. 1. Observe that for both controllers obtained through the proposed design method, the control objective is achieved with $\left|u_{i}(t)\right|<U_{i}, i=1,2, \forall t \geq 0$. On the contrary, the rolling moment $u_{2}$ generated by the algorithm of [Frye et al.(2010)] takes absolute values greater than $U_{2}=10$ during the transient; in a bounded input context, this controller would have undergone input saturation. It is important to note that the boundedness of the proposed scheme restricts the closed loop response speed, which is a natural consequence of the power supply limitations of constrained inputs. This is corroborated through Fig. 1 whence one perceives the contrast among the velocity estimation error vanishing, achieved in around 10 seconds via the considered finite-time observer with unbounded dynamics, and the position stabilization completed in almost 50 seconds in view of the considered initial conditions (with a considerable vertically upwards initial impulsion). Long stabilization times have been characteristic of bounded approaches in the face of demanding initial conditions even in state-feedback contexts [Ye et al.(2007)], [Zavala-Río et al.(2003)]. The longer stabilization arisen through the algorithm of [Frye et al.(2010)] is rather a consequence of the controller structure in view of the expressions used for the auxiliary (input) variables $v_{1}$ and $v_{2}$ - denoted $r_{1}$ and $r_{2}$ in [Frye et al.(2010)] -, which become very small when (at least) one of the system states get considerably high values during the transient which causes the horizontal or vertical motion closed-loop dynamics to become very slow. Let us further note that with respect to the performance obtained through controller 1, a shortest stabilization time takes place with controller 2, which is particularly seen through the horizontal position responses; such performance improvement corroborates the usefulness of the freedom to adjust the controller structure furnished through the generalized versions of the involved finite-time stabilizers — which is more significant in (but not exclusive to) the case of that of Lemma 4, directly used in the design of $u_{2}$ in (18)- 

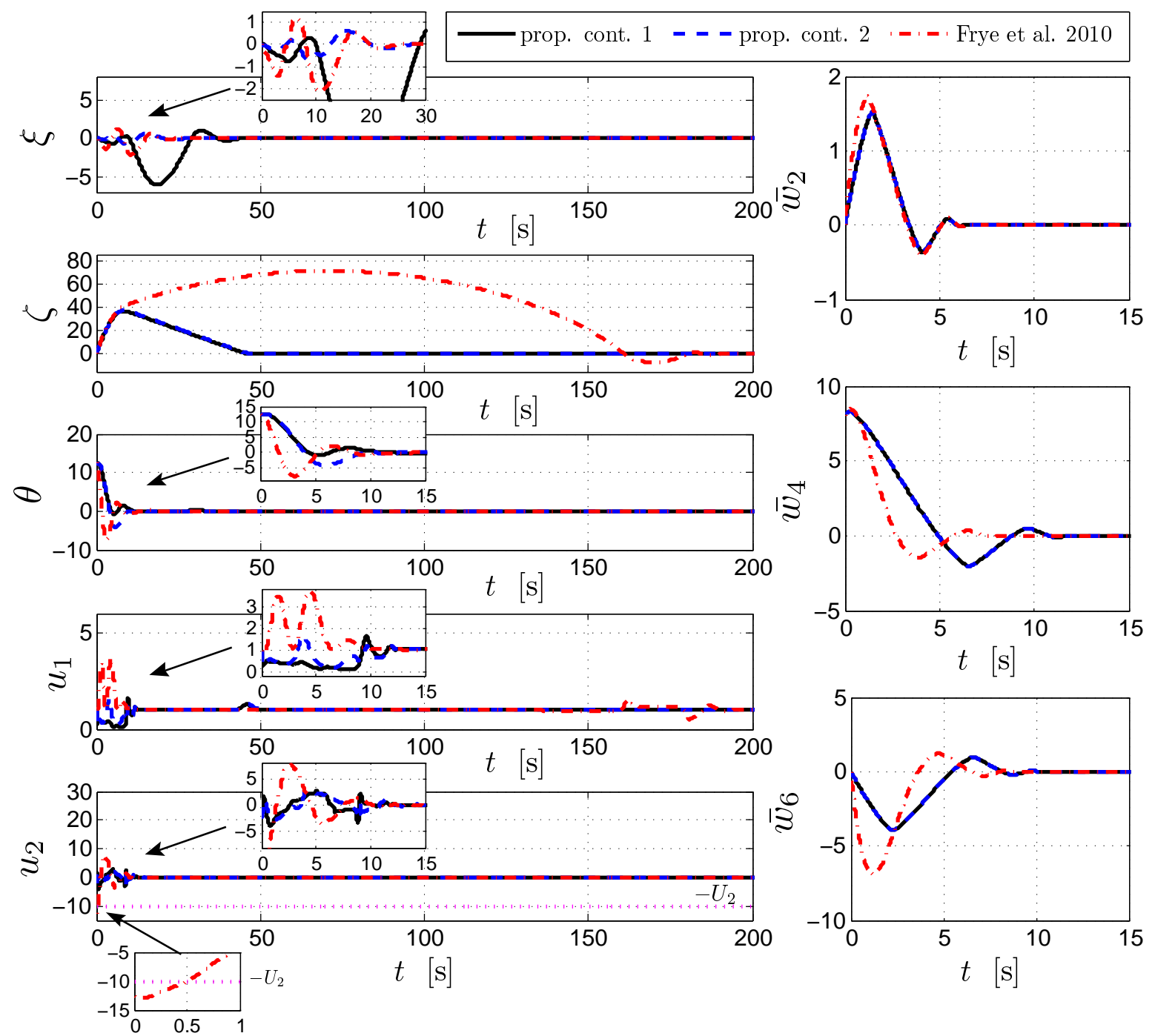

Fig. 1. Simulation results. Left: position responses $(\xi, \zeta, \theta)$ and control signals $\left(u_{1}, u_{2}\right)$. Right: velocity estimation errors $\left(\bar{w}_{i}, i=2,4,6\right)$.

\section{EXPERIMENTAL RESULTS}

Experimental implementation of the developed algorithm was an important challenge of the present work. Realization of a PVTOL-type task on an actual unmanned aerial vehicle (UAV) has been stated to be the main goal of the test. The closed-loop performance could hardly be expected to look like that perceived in Section VI in the face of structural differences with respect to a PVTOL device or technical inaccuracies. Indeed, it is important to take into account that the analysis and synthesis developed in the previous sections did not include perturbation terms in the system dynamics or measurement imprecisions such as delays. However, the test itself (whether the results are close to or far from ideal ones) would be helpful to evaluate the implementability of the algorithm and the closed-loop performance under model and/or technical discrepancies.

The experimental UAV where the proposed scheme was implemented on, shown in Fig. 2, is a quadrotor based on the Mikrokopter frame (but with modifications). It has four brushless motors driven with BLCTRLV2 controllers. The total weight is $1.2 \mathrm{~kg}$, using a $11.1 \mathrm{~V} \mathrm{LiPO}$ battery of $6000 \mathrm{mAh}$, giving about 15 minutes of flight time. The main electronic board is based on an IGEP module, equipped with a System On Chip (SOC) DM3730 from Texas Instruments. This SOC has one ARM Cortex $A 8$ core running at $1 \mathrm{GHz}$ and one DSP C64x+ core running at $800 \mathrm{MHz}$ (which allows embedded image processing, but this is not used in our application). The ARM processor allows to run Linux and its real-time extension Xenomai. Thus, the control law runs in real time at $100 \mathrm{~Hz}$. The UAV is also equipped with a Microstrain 3DMGX3-25 IMU giving Euler angles and rotation speed measurements at $100 \mathrm{~Hz}$. In order to measure the UAV position we used an external sensor, namely a motion capture system. More specifically, we used an Optitrack system composed of 12 cameras fixed in a $5 \mathrm{~m} \times 7 \mathrm{~m}$ room. All cameras are linked to a server computing the UAV position in turn delivered through a wireless connection (XBee modem). This system is able to deliver position at up to $250 \mathrm{~Hz}$, but in our experiments the frame rate was fixed at $100 \mathrm{~Hz}$. Higher frame rates are not useful because of the delay introduced through the wireless connection. Let us note that the motion capture 


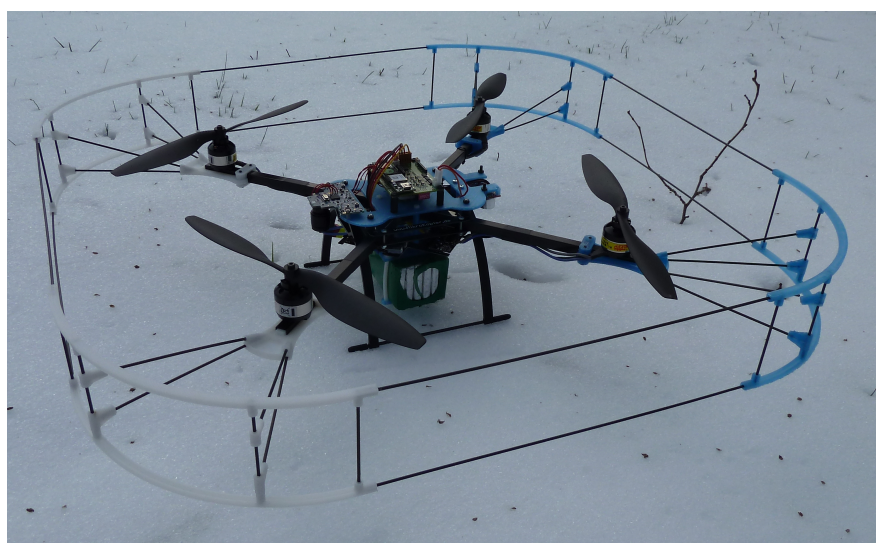

Fig. 2. Experimental quadrotor

system does not deliver translational velocity measurements. The only speed measurements available are the angular ones, through the IMU.

The quadrotor may be seen as two coupled PVTOL aircraft, each of them at every one of its axes. This is suitably illustrated through a thorough technical description in [Lozano et al.(2004)]. Moreover, [Castillo et al.(2004)] shows that, by keeping a motionless yaw angle at zero, the quadrotor dynamics becomes equivalent to that of a PVTOL aircraft under a motionless zero roll angle, with the depth position and pitch angle characterizing the horizontal and angular displacements (on the corresponding plane) $-\xi$ and $\theta-$ under the described conditions, while a similar PVTOL aircraft dynamics is obtained under a motionless zero pitch angle, with the lateral position and roll angle characterizing $\xi$ and $\theta$ in this latter case. In view of this, one of the axis of the experimental quadrotor considered here was held stabilized - so as to keep motionless zero depth displacements and pitch rotations - through the previously-tested state-feedback controller presented in [Sanahuja et al.(2010)]. The remaining axis moved on its corresponding plane with a behavior similar to that of a PVTOL aircraft (as shown in [Castillo et al.(2004)] and described in [Lozano et al.(2004)]). It was on this axis that the proposed approach was tested.

The stabilization scheme was implemented taking $\varepsilon=0$ in the control expressions, (14)-(21), and adopting the structure of both controllers 1 and 2 of the precedent section. The functions $\sigma_{0}(s), \sigma_{i j}(s), i, j=1,2, \sigma_{3 l}(s), l=0, \ldots, 3$, and $\sigma_{m n}(s)$, $m=4,5,6, n=1,2$, were defined as correspondingly specified in Appendix $H$. The parameter and gain values were fixed - under the consideration of conditions (21) and (22) - (as in the previous section, units will be omitted for the sake of simplicity) as: $M_{11}=0.5, M_{21}=1.3, M_{22}^{-}=M_{22}^{+}=0.8, M_{30}=M_{31}=2, \beta_{33}=1, \beta_{41}=\beta_{51}=0.9, \beta_{42}=\beta_{52}=0.8, \beta_{61}=0.99$, $\beta_{62}=0.98, k_{11}=k_{22}=4.9, k_{21}=5.9, k_{31}=3, k_{32}=0.15, k_{41}=k_{51}=490.5, k_{61}=20, k_{42}=k_{52}=4905$ and $k_{62}=500$ for both controllers, while: $M_{32}=20, L_{32}=0.5, M_{11}=0.5, k_{0}=0.4$ and $k_{12}=4.9$ for controller 1 , and: $M_{33}=27, L_{33}=0.75$, $M_{11}=0.7, k_{0}=0.3$ and $k_{12}=2.94$ for controller 2 . The initial conditions for all the estimation variables were fixed at zero, i.e. $\hat{w}_{i}(0)=0, \forall i=1, \ldots, 6$. The values of $\xi_{d}$ and $\zeta_{d}$ were fixed such that stabilization was performed towards horizontal and vertical target positions of $1 \mathrm{~m}$ and $0.4 \mathrm{~m}$ respectively. The test was implemented with the quadrotor initially close to the home (reference) location.

Results are shown in Fig. 3. The stabilization objective is observed to be achieved by both controllers. In particular, the rolling moment control signal generated by controller 1 is noticed to be oscillating during the transient, having a slight (vanishing) effect on the angular position response. There are two main arguments for this phenomenon. The model and technical inaccuracies, on the one hand, may give rise to important position-error and velocity-error control actions in $u_{2}$ during the transient. On the other hand, by directly saturating each of the referred control actions in $u_{2}$, controller 1 considerably limits its ability to cope with important initial errors; by rather bounding the addition of both referred terms, controller 2 avoids a direct saturation on the velocity-error action and releases the saturation bound on the position-error action from design constraints, permitting more suitable reactions to such errors. Controller 1 may consequently be concluded to be more sensitive to important initial errors as well as model and technical inaccuracies, while controller 2 proves to overcome such limitation. It is worth pointing out that the effects related to model and technical imprecisions have been present in experimental results shown in previous works [Lozano et al.(2004)], [Sanahuja et al.(2010)], [Lopez-Araujo et al.(2010)], where oscillating angular position responses have generally arisen even under the availability of all the system variables considered by such state-feedback approaches.

\section{CONCLUSIONS}

In this work, an output feedback scheme for the global stabilization of the PVTOL aircraft with bounded inputs has been developed taking into account the positive nature of the thrust. With respect to previous approaches, the proposed controller guarantees the global stabilization objective disregarding velocity measurements simultaneously avoiding input saturation. To deal with the lack of velocity measurements, the proposed algorithm involves a finite-time observer. Finite-time roll-angle 

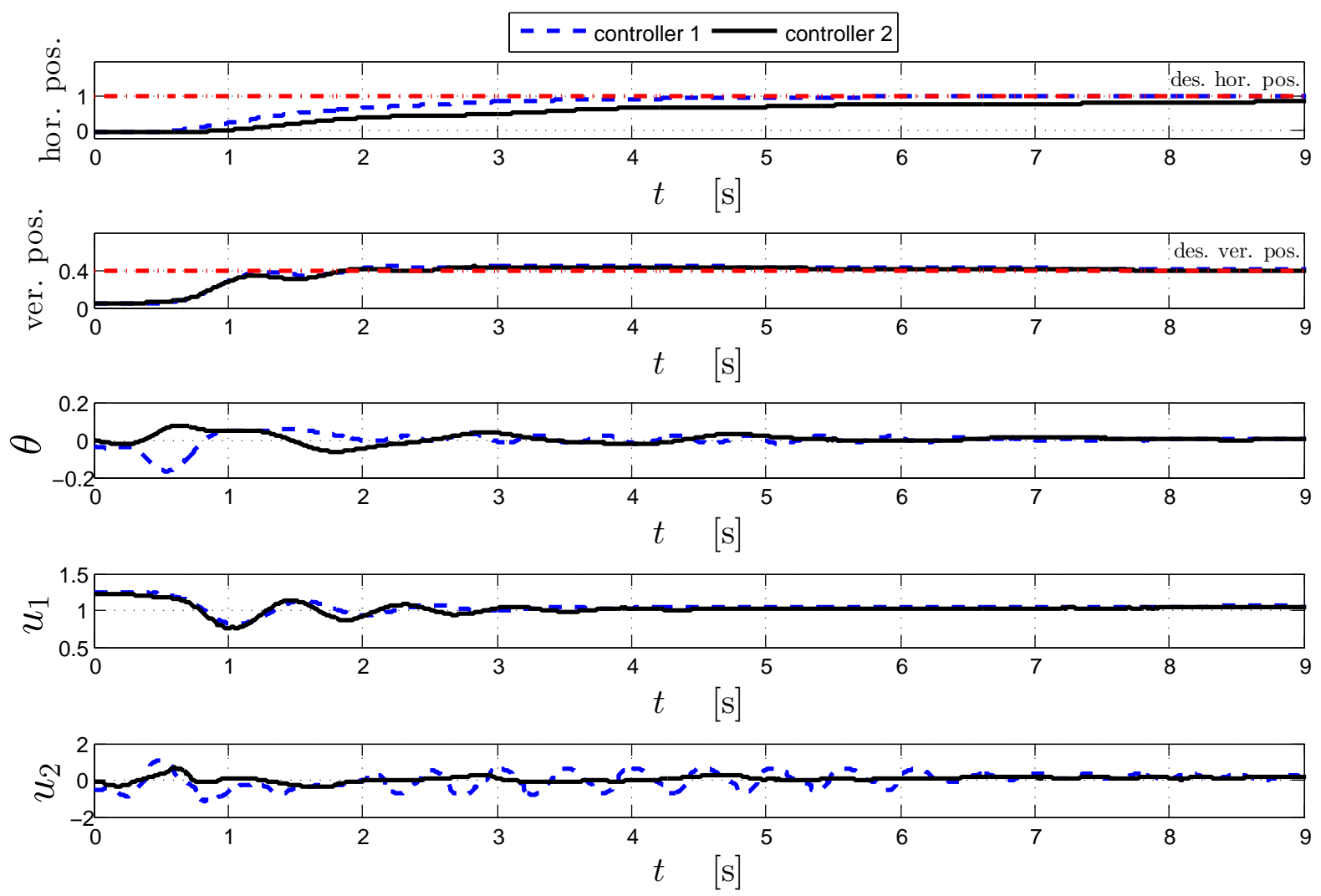

Fig. 3. Experimental results: position responses (horizontal, vertical, $\theta)$ and control signals $\left(u_{1}, u_{2}\right)$.

tracking is simultaneously performed to achieve the stabilization objective in the underactuated natural context of the PVTOL aircraft. Successful simulation results have confirmed the efficiency of the proposed scheme, as well as the usefulness for performance improvement purposes - of the freedom to adjust the controller structure furnished through the generalized versions of the involved finite-time stabilizers. Achievement of the stabilization objective was further corroborated through experimental tests on an actual UAV system despite model and technical discrepancies. The effects of such inaccuracies, generally observed in actual applications, may be expected to be improved by considering perturbation terms and/or technical imprecisions (such as measurement delays) in the system dynamics. A formal work in that direction as well as the extension of the techniques developed/involved in this paper to UAV's with more complex dynamics (such as quadrotor aircraft) constitute potential topics for future research.

\section{APPENDIX A \\ PROOF OF LEMMA 1}

For any $j \in \mathbb{Z}_{m}^{+}$, let $\sigma_{\infty}^{(j)}=\lim _{|s| \rightarrow \infty} \sigma^{(j)}(s)$.

1) a) $p=0$ :

i) $q=1$ :

Observe that since $\sigma$ is a strictly passive function that is nondecreasing and bounded by $M$, there exist positive constants $c^{-} \leq M$ and $c^{+} \leq M$ such that

$$
\lim _{|s| \rightarrow \infty} \sigma(s)=\frac{(\operatorname{sign}(s)-1) c^{-}+(\operatorname{sign}(s)+1) c^{+}}{2}=\sigma_{\infty}
$$


Hence,

$$
\begin{aligned}
\lim _{|s| \rightarrow \infty} \sigma^{\prime}(s) & =\lim _{|s| \rightarrow \infty} \lim _{h \rightarrow 0} \frac{\sigma(s+h)-\sigma(s)}{h} \\
& =\lim _{h \rightarrow 0} \lim _{|s| \rightarrow \infty} \frac{\sigma(s+h)-\sigma(s)}{h} \\
& =\lim _{h \rightarrow 0} \frac{\sigma_{\infty}-\sigma_{\infty}}{h}=0
\end{aligned}
$$

ii) Suppose that $m \geq 2$ and let $q \in \mathbb{N}_{m} \backslash\{1\}$. Observe that

$$
\begin{aligned}
\lim _{|s| \rightarrow \infty} \sigma^{(q)}(s) & =\lim _{|s| \rightarrow \infty} \lim _{h \rightarrow 0} \frac{\sigma^{(q-1)}(s+h)-\sigma^{(q-1)}(s)}{h} \\
& =\lim _{h \rightarrow 0} \lim _{|s| \rightarrow \infty} \frac{\sigma^{(q-1)}(s+h)-\sigma^{(q-1)}(s)}{h} \\
& =\lim _{h \rightarrow 0} \frac{1}{h}\left[\lim _{|s| \rightarrow \infty} \sigma^{(q-1)}(s+h)-\lim _{|s| \rightarrow \infty} \sigma^{(q-1)}(s)\right]
\end{aligned}
$$

In view of the result of point 1(a)i of the proof, by analyzing this expression in order from $q=2$ to $q=m$, one sees that $\sigma^{(q-1)}(s+h) \rightarrow \sigma^{(q-1)}(s) \rightarrow 0$ as $|s| \rightarrow \infty, \forall q \in \mathbb{N}_{m} \backslash\{1\}$, and consequently $\lim _{|s| \rightarrow \infty} \sigma^{(q)}(s)=0$, $\forall q \in \mathbb{N}_{m} \backslash\{1\}$.

b) $p \geq 1$ :

Observe that $s^{p} \sigma^{(q-1)}(s) \rightarrow s^{p} \sigma_{\infty}^{(q-1)}$ as $|s| \rightarrow \infty$ where, according to point 1a of the proof, $\sigma_{\infty}^{(q-1)}$ is a well-defined finite value for all $(q-1) \in \mathbb{Z}_{m}^{+}$. Then, $\frac{d}{d s} s^{p} \sigma^{(q-1)}(s) \rightarrow \frac{d}{d s} s^{p} \sigma_{\infty}^{(q-1)}=p s^{p-1} \sigma_{\infty}^{(q-1)}$ as $|s| \rightarrow \infty$. Consequently,

$$
\begin{aligned}
\lim _{|s| \rightarrow \infty} \frac{d}{d s} s^{p} \sigma^{(q-1)}(s) & =\lim _{|s| \rightarrow \infty}\left(p s^{p-1} \sigma^{(q-1)}(s)+s^{p} \sigma^{(q)}(s)\right) \\
& =\lim _{|s| \rightarrow \infty} p s^{p-1} \sigma_{\infty}^{(q-1)}+\lim _{|s| \rightarrow \infty} s^{p} \sigma^{(q)}(s)=\lim _{|s| \rightarrow \infty} p s^{p-1} \sigma_{\infty}^{(q-1)}
\end{aligned}
$$

wherefrom one sees that $\lim _{|s| \rightarrow \infty} s^{p} \sigma^{(q)}(s)=0, \forall p \geq 1, \forall q \in \mathbb{N}_{m}$.

2) Note that, in view of its continuity, $s^{p} \sigma^{(q)}(s)$ is bounded on any compact subset of $\mathbb{R}$. Thus, its boundedness holds on $\mathbb{R}$ if $\lim _{|s| \rightarrow \infty}\left|s^{p} \sigma^{(q)}(s)\right|<\infty$. Since, according to item 1 of the statement, $\lim _{|s| \rightarrow \infty} s^{p} \sigma^{(q)}(s)=0, \forall p \in \mathbb{Z}^{+}, \forall q \in \mathbb{N}_{m}$, we conclude that, for all $p \in \mathbb{Z}_{+}$and all $q \in \mathbb{N}_{m}$, there exist $A^{p, q} \in(0, \infty)$ such that $\left|s^{p} \sigma^{(q)}(s)\right| \leq A^{p, q}, \forall s \in \mathbb{R}$.

\section{APPENDIX B}

\section{PROOF OF LEMMA 2}

1) Note that in view of the analytical properties that define a strongly passive function, we have that

$$
\begin{aligned}
\int_{0}^{s} \sigma(k v) d v \geq \int_{0}^{s} \operatorname{sign}(v) \kappa|a \operatorname{sat}(k v / a)|^{\alpha} d v & \\
& =\int_{0}^{s} \operatorname{sign}(v) \kappa(\min \{|k v|, a\})^{\alpha} d v \\
& \triangleq \Sigma(s)= \begin{cases}\frac{\kappa k^{\alpha}}{\alpha+1}|s|^{\alpha+1} & \forall|s| \leq \frac{a}{k} \\
\kappa a^{\alpha}\left(|s|-\frac{a \alpha}{k(\alpha+1)}\right) & \forall|s|>\frac{a}{k}\end{cases}
\end{aligned}
$$

From this expression one sees that $\underline{\Sigma}(s)>0, \forall s \neq 0$, and consequently $\int_{0}^{s} \sigma(k v) d v>0, \forall s \neq 0$.

2) Notice from (39) that $\underline{\Sigma}(s) \rightarrow \infty$ as $|s| \rightarrow \infty$ and consequently $\int_{0}^{s} \sigma(k v) d v \rightarrow \infty$ as $|s| \rightarrow \infty$.

3) Observe that since $\sigma_{1}$ and $\sigma_{2}$ are strictly passive, we have that

$$
(\forall s \neq 0)\left(s \sigma_{i}(s)>0\right) \Longleftrightarrow \operatorname{sign}\left(\sigma_{i}(s)\right)=\operatorname{sign}(s)
$$

$\forall i \in \mathbb{N}_{2}$. Then,

$$
\operatorname{sign}\left(\sigma_{1}\left(\sigma_{2}(s)\right)\right)=\operatorname{sign}\left(\sigma_{2}(s)\right)=\operatorname{sign}(s) \Longleftrightarrow(\forall s \neq 0)\left(s \sigma_{1}\left(\sigma_{2}(s)\right)>0\right)
$$


whence one sees that $\sigma_{1} \circ \sigma_{2}$ is strictly passive. On the other hand, since there are positive constants $\kappa_{i}, \alpha_{i}$, and $a_{i}$ such that $(\forall s \in \mathbb{R})\left(\left|\sigma_{i}(s)\right| \geq \kappa_{i}\left|a_{i} \operatorname{sat}\left(s / a_{i}\right)\right|^{\alpha_{i}}\right), \forall i \in \mathbb{N}_{2}$, then

$$
\begin{gathered}
\left|\sigma_{1}\left(\sigma_{2}(s)\right)\right| \geq \kappa_{1}\left|a_{1} \operatorname{sat}\left(\sigma_{2}(s) / a_{1}\right)\right|^{\alpha_{1}}=\kappa_{1}\left(\min \left\{\left|\sigma_{2}(s)\right|, a_{1}\right\}\right)^{\alpha_{1}} \\
\geq \min \left\{\kappa_{1}\left(\kappa_{2}\left|a_{2} \operatorname{sat}\left(s / a_{2}\right)\right|^{\alpha_{2}}\right)^{\alpha_{1}}, \kappa_{1} a_{1}^{\alpha_{1}}\right\} \\
=\min \left\{\kappa_{1} \kappa_{2}^{\alpha_{1}}\left(\min \left\{|s|, a_{2}\right\}\right)^{\alpha_{1} \alpha_{2}}, \kappa_{1} a_{1}^{\alpha_{1}}\right\} \\
=\min \left\{\kappa_{1} \kappa_{2}^{\alpha_{1}}|s|^{\alpha_{1} \alpha_{2}}, \kappa_{1}\left(\min \left\{\kappa_{2} a_{2}^{\alpha_{2}}, a_{1}\right\}\right)^{\alpha_{1}}\right\} \\
\geq \kappa_{\circ}\left(\min \left\{|s|, a_{\circ}\right\}\right)^{\alpha_{\circ}}=\kappa_{\circ}\left|a_{\circ} \operatorname{sat}\left(s / a_{\circ}\right)\right|^{\alpha_{\circ}}
\end{gathered}
$$

$\forall s \in \mathbb{R}$, for positive constants

$$
\kappa_{\circ} \leq \kappa_{1} \kappa_{2}^{\alpha_{1}}, \quad \alpha_{\circ} \geq \alpha_{1} \alpha_{2}, \quad \text { and } \quad a_{\circ} \leq\left(\frac{\min \left\{\kappa_{2} a_{2}^{\alpha_{2}}, a_{1}\right\}}{\kappa_{2}}\right)^{1 / \alpha_{2}}
$$

Thus $\alpha_{1} \circ \alpha_{2}$ is strongly passive.

\section{APPENDiX C}

\section{PROOF OF LEMMA 3}

Let $s_{1}, s_{2}, s_{3} \in \mathbb{R}$. Since $\sigma_{0}$ is strictly increasing, we have that $\sigma_{0}\left(s_{3}\right)>\sigma_{0}\left(s_{2}\right) \Longleftrightarrow s_{3}>s_{2}$ and $\sigma_{0}\left(s_{3}\right)<\sigma_{0}\left(s_{2}\right) \Longleftrightarrow s_{3}<s_{2}$. From this and the strictly passive character of $\sigma_{1}$ we have, by letting $s_{3}=\sigma_{1}\left(k s_{1}\right)+s_{2}$, that $\sigma_{0}\left(\sigma_{1}\left(k s_{1}\right)+s_{2}\right)-\sigma_{0}\left(s_{2}\right)>0 \Longleftrightarrow$ $\sigma_{1}\left(k s_{1}\right)>0 \Longleftrightarrow s_{1}>0$ and $\sigma_{0}\left(\sigma_{1}\left(k s_{1}\right)+s_{2}\right)-\sigma_{0}\left(s_{2}\right)<0 \Longleftrightarrow \sigma_{1}\left(k s_{1}\right)<0 \Longleftrightarrow s_{1}<0, \forall s_{2} \in \mathbb{R}$, whence it follows that $s_{1}\left[\sigma_{0}\left(\sigma_{1}\left(k s_{1}\right)+s_{2}\right)-\sigma_{0}\left(s_{2}\right)\right]>0, \forall s_{1} \neq 0, \forall s_{2} \in \mathbb{R}$.

\section{APPENDIX D}

\section{PROOF OF LEMMA 4}

Let us define the continuously differentiable scalar function

$$
V_{1}\left(x_{1}, x_{2}\right)=\frac{x_{2}^{2}}{2}+\int_{0}^{x_{1}} \sigma_{3}\left(\sigma_{1}\left(k_{1} s\right)\right) d s
$$

From Remark 2 and Lemma 2 one sees that $V_{1}\left(x_{1}, x_{2}\right)$ is a positive definite radially unbounded function. Its derivative along the trajectories of system (8) is given by

$$
\begin{aligned}
\dot{V}_{1}\left(x_{1}, x_{2}\right) & =x_{2} \dot{x}_{2}+\sigma_{3}\left(\sigma_{1}\left(k_{1} x_{1}\right)\right) \dot{x}_{1} \\
& =-x_{2} \sigma_{3}\left(\sigma_{1}\left(k_{1} x_{1}\right)+\sigma_{2}\left(k_{2} x_{2}\right)\right)+\sigma_{3}\left(\sigma_{1}\left(k_{1} x_{1}\right)\right) x_{2} \\
& =-x_{2}\left[\sigma_{3}\left(\sigma_{1}\left(k_{1} x_{1}\right)+\sigma_{2}\left(k_{2} x_{2}\right)\right)-\sigma_{3}\left(\sigma_{1}\left(k_{1} x_{1}\right)\right)\right]
\end{aligned}
$$

From Lemma 3, one sees that $\dot{V}_{1}\left(x_{1}, x_{2}\right) \leq 0, \forall\left(x_{1}, x_{2}\right) \in \mathbb{R}^{2}$, with $\dot{V}_{1}\left(x_{1}, x_{2}\right)=0 \Longleftrightarrow x_{2}=0$. Further, from the system dynamics in Eqs. (8), taking into account that $\sigma_{i}, i=1,2,3$, are strictly passive, one sees that $x_{2}(t) \equiv 0 \Longrightarrow \dot{x}_{2}(t) \equiv$ $0 \Longrightarrow \sigma_{3}\left(\sigma_{1}\left(k_{1} x_{1}(t)\right)\right) \equiv 0 \Longrightarrow x_{1}(t) \equiv 0$. Then, by the Invariance Theory [Michel et al.(2008), §7.2] (more specifically, by [Michel et al.(2008), Corollary 7.2.1]), one concludes that $(0,0)$ is a globally asymptotically stable equilibrium. Let us now additionally suppose that, for every $i=1,2, \sigma_{i}(s)$ is locally $r_{i}$-homogeneous of degree $\alpha$, with domain of homogeneity $D_{i}=\left\{s \in \mathbb{R}:|s|<\rho_{i} \in(0, \infty]\right\}$, and $\sigma_{3}(s)$ is locally $\alpha$-homogeneous of degree $\alpha_{3}$ with domain of homogeneity $D_{3}=\left\{s \in \mathbb{R}:|s|<\rho_{3} \in(0, \infty]\right\}$, for some dilation coefficients such that (9) is satisfied. Let $x=\left(x_{1}, x_{2}\right)^{T}, D=\left\{x \in \mathbb{R}^{2}:\left|x_{1}\right|<\right.$ $\left.\rho_{1},\left|x_{2}\right|<\rho_{2},\left|\sigma_{1}\left(k_{1} x_{1}\right)+\sigma_{2}\left(k_{2} x_{2}\right)\right|<\rho_{3}\right\}, r \triangleq\left(r_{1}, r_{2}\right)$, and $f(x) \triangleq\left(f_{1}(x), f_{2}(x)\right)^{T}$. Observe that for every $x \in D$ and all $\varepsilon \in \mathbb{R}_{>0}$ such that $\delta_{\varepsilon}^{r}(x) \in D$, we have that

$$
\begin{aligned}
f_{1}\left(\varepsilon^{r_{1}} x_{1}, \varepsilon^{r_{2}} x_{2}\right) & =\varepsilon^{r_{2}} x_{2} \\
& =\varepsilon^{r_{2}-r_{1}+r_{1}} x_{2} \\
& =\varepsilon^{\left(r_{2}-r_{1}\right)+r_{1}} f_{1}\left(x_{1}, x_{2}\right)
\end{aligned}
$$

and

$$
\begin{aligned}
f_{2}\left(\varepsilon^{r_{1}} x_{1}, \varepsilon^{r_{2}} x_{2}\right) & =-\sigma_{3}\left(\sigma_{1}\left(k_{1} \varepsilon^{r_{1}} x_{1}\right)+\sigma_{2}\left(k_{2} \varepsilon^{r_{2}} x_{2}\right)\right) \\
& =-\sigma_{3}\left(\varepsilon^{\alpha} \sigma_{1}\left(k_{1} x_{1}\right)+\varepsilon^{\alpha} \sigma_{2}\left(k_{2} x_{2}\right)\right) \\
& =-\varepsilon^{\alpha_{3}} \sigma_{3}\left(\sigma_{1}\left(k_{1} x_{1}\right)+\sigma_{2}\left(k_{2} x_{2}\right)\right) \\
& =-\varepsilon^{2 r_{2}-r_{1}} \sigma_{3}\left(\sigma_{1}\left(k_{1} x_{1}\right)+\sigma_{2}\left(k_{2} x_{2}\right)\right) \\
& =\varepsilon^{\left(r_{2}-r_{1}\right)+r_{2}} f_{2}\left(x_{1}, x_{2}\right)
\end{aligned}
$$


Thus, $f(x)$ is locally $r$-homogeneous — with domain of homogeneity $D$ - of degree $k=r_{2}-r_{1}<0$, and consequently, according to Theorem $1,(0,0)$ is globally finite-time stable.

\section{APPENDIX E}

PROOF OF COROLLARY 1

Let us begin by noting that, for every $i \in \mathbb{N}_{3}$ :

$$
\begin{aligned}
\sigma_{i}\left(\varepsilon^{r_{i}} s\right) & =\kappa_{i} \operatorname{sign}\left(\varepsilon^{r_{i}} s\right)\left|\varepsilon^{r_{i}} s\right|^{\beta_{i}} \\
& =\varepsilon^{r_{i} \beta_{i}} \kappa_{i} \operatorname{sign}(s)|s|^{\beta_{i}} \\
& =\varepsilon^{r_{i} \beta_{i}} \sigma_{i}(s)
\end{aligned}
$$

for any $r_{i}>0$, every $s \in D_{i}$, and all $\varepsilon>0$ such that $\varepsilon^{r_{i}} \in D_{i}$. Hence, for any $\left(r_{1}, r_{2}, r_{3}\right) \in \mathbb{R}_{>0}^{3}$ such that $r_{3}=r_{1} \beta_{1}=r_{2} \beta_{2}=\alpha$, one sees on the one hand that, for every $i \in \mathbb{N}_{2}, \sigma_{i}(s)$ is locally $r_{i}$-homogeneous of degree $\alpha$, and $\sigma_{3}(s)$ is locally $\alpha$-homogeneous of degree $\alpha_{3}=\alpha \beta_{3}$, while it follows that

$$
\begin{aligned}
\beta_{3}=\frac{2}{\beta_{2}}-\frac{1}{\beta_{1}}>0>\frac{1}{\beta_{2}}-\frac{1}{\beta_{1}} & \Longleftrightarrow \alpha \beta_{3}=\frac{2 \alpha}{\beta_{2}}-\frac{\alpha}{\beta_{1}}>0>\frac{\alpha}{\beta_{2}}-\frac{\alpha}{\beta_{1}} \\
& \Longleftrightarrow \alpha_{3}=2 r_{2}-r_{1}>0>r_{2}-r_{1}
\end{aligned}
$$

\section{APPENDIX F}

PROOF OF LEMMA 5

Let us define the continuously differentiable scalar function

$$
V_{2}\left(x_{1}, x_{2}\right)=\frac{x_{2}^{2}}{2}+\int_{0}^{x_{1}} \sigma_{2}\left(k_{2} s\right) d s
$$

Under the consideration of items 1 and 2 of Lemma 2, one sees that $V_{2}\left(x_{1}, x_{2}\right)$ is a positive definite radially unbounded function. Its derivative along the trajectories of system (11) is given by

$$
\begin{aligned}
\dot{V}_{2}\left(x_{1}, x_{2}\right) & =x_{2} \dot{x}_{2}+\sigma_{2}\left(k_{2} x_{1}\right) \dot{x}_{1} \\
& =-x_{2} \sigma_{2}\left(k_{2} x_{1}\right)+\sigma_{2}\left(k_{2} x_{1}\right)\left[x_{2}-\sigma_{1}\left(k_{1} x_{1}\right)\right] \\
& =-\sigma_{1}\left(k_{1} x_{1}\right) \sigma_{2}\left(k_{2} x_{1}\right)
\end{aligned}
$$

Since $\sigma_{1}$ and $\sigma_{2}$ are strictly passive, one sees that $\dot{V}_{2}\left(x_{1}, x_{2}\right) \leq 0, \forall\left(x_{1}, x_{2}\right) \in \mathbb{R}^{2}$, with $\dot{V}_{2}\left(x_{1}, x_{2}\right)=0 \Longleftrightarrow x_{1}=0$. Further, from the system dynamics in Eqs. (11), taking into account that $\sigma_{1}$ and $\sigma_{2}$ are strictly passive, one sees that $x_{1}(t) \equiv 0 \Longrightarrow$ $\left(\dot{x}_{1}(t) \equiv 0\right) \wedge\left(\sigma_{1}\left(k_{1} x_{1}(t)\right) \equiv 0\right) \Longrightarrow x_{2}(t) \equiv 0$. Then, by the Invariance Theory [Michel et al.(2008), §7.2], one concludes that $(0,0)$ is a globally asymptotically stable equilibrium. Let us now additionally suppose that, for every $i \in \mathbb{N}_{2}, \sigma_{i}(s)$ is locally $r_{0}$-homogeneous of degree $\alpha_{i}$, with domain of homogeneity $D_{i}=\left\{s \in \mathbb{R}:|s|<\rho_{i} \in(0, \infty]\right\}$, for some (common) dilation coefficient such that (12) is satisfied. Let $x=\left(x_{1}, x_{2}\right)^{T}, D=\left\{x \in \mathbb{R}^{2}:\left|x_{i}\right|<\rho_{i}, \forall i \in \mathbb{N}_{2}\right\}, r=\left(r_{0}, \alpha_{1}\right)$, and $f(x)=\left(f_{1}(x), f_{2}(x)\right)^{T}$. Observe that for every $x \in D$ and all $\varepsilon \in \mathbb{R}_{>0}$ such that $\delta_{\varepsilon}^{r}(x) \in D$, we have that

$$
\begin{aligned}
f_{1}\left(\varepsilon^{r} x_{1}, \varepsilon^{\alpha_{1}} x_{2}\right) & =\varepsilon^{\alpha_{1}} x_{2}-\sigma_{1}\left(k_{1} \varepsilon^{r} x_{1}\right) \\
& =\varepsilon^{\alpha_{1}} x_{2}-\varepsilon^{\alpha_{1}} \sigma_{1}\left(k_{1} x_{1}\right) \\
& =\varepsilon^{\alpha_{1}-r+r}\left[x_{2}-\sigma_{1}\left(k_{1} x_{1}\right)\right] \\
& =\varepsilon^{\left(\alpha_{1}-r\right)+r} f_{1}\left(x_{1}, x_{2}\right)
\end{aligned}
$$

and

$$
\begin{aligned}
f_{2}\left(\varepsilon^{r} x_{1}, \varepsilon^{\alpha_{1}} x_{2}\right) & =-\sigma_{2}\left(k_{2} \varepsilon^{r} x_{1}\right) \\
& =-\varepsilon^{\alpha_{2}} \sigma_{2}\left(k_{2} x_{1}\right) \\
& =-\varepsilon^{2 \alpha_{1}-r} \sigma_{2}\left(k_{2} x_{1}\right) \\
& =\varepsilon^{\left(\alpha_{1}-r\right)+\alpha_{1}} f_{2}\left(x_{1}, x_{2}\right)
\end{aligned}
$$

Thus, $f(x)$ is locally $r$-homogeneous — with domain of homogeneity $D$ - of degree $k=\alpha_{1}-r<0$, and consequently, according to Theorem $1,(0,0)$ is globally finite-time stable. 


\section{APPENDIX G}

PROOF OF COROLLARY 2

Let us begin by noting that, for every $i \in \mathbb{N}_{2}$ :

$$
\begin{aligned}
\sigma_{i}\left(\varepsilon^{r_{0}} s\right) & =\kappa_{i} \operatorname{sign}\left(\varepsilon^{r_{0}} s\right)\left|\varepsilon^{r_{0}} s\right|^{\beta_{i}} \\
& =\varepsilon^{r_{0} \beta_{i}} \kappa_{i} \operatorname{sign}(s)|s|^{\beta_{i}} \\
& =\varepsilon^{r_{0} \beta_{i}} \sigma_{i}(s)
\end{aligned}
$$

for any $r_{0}>0$, every $s \in D_{i}$, and all $\varepsilon>0$ such that $\varepsilon^{r_{0}} s \in D_{i}$. Hence, for any $r_{0}>0$, one sees that $\sigma_{i}(s), i=1,2$, are locally $r_{0}$-homogeneous of degree $\alpha_{i}=r_{0} \beta_{i}$, whence one further corroborates that

$$
\begin{aligned}
\beta_{2}=2 \beta_{1}-1>0>\beta_{1}-1 & \Longleftrightarrow r_{0} \beta_{2}=2 r_{0} \beta_{1}-r_{0}>0>r_{0} \beta_{1}-r_{0} \\
& \Longleftrightarrow \alpha_{2}=2 \alpha_{1}-r_{0}>0>\alpha_{1}-r_{0}
\end{aligned}
$$

\section{APPENDIX H}

FUNCTIONS INVOLVED IN THE SIMULATION TESTS

Let

$$
\sigma_{u}\left(s ; \beta_{u}\right) \triangleq \operatorname{sign}(s)|s|^{\beta_{u}}
$$

and

$$
\sigma_{b}\left(s ; \beta_{b}, L_{b}, M_{b}\right) \triangleq \begin{cases}\operatorname{sign}(s) \frac{L_{b}^{1-\beta_{b}}}{\beta_{b}}|s|^{\beta_{b}} & \forall|s|<L_{b} \\ \operatorname{sign}(s) \frac{L_{b}}{\beta_{b}}+\left(M_{b}-\frac{L_{b}}{\beta_{b}}\right) \tanh \left(\frac{s-\operatorname{sign}(s) L_{b}}{M_{b}-L_{b} / \beta_{b}}\right) & \forall|s| \geq L_{b}\end{cases}
$$

with $L_{b}<\beta_{b} M_{b}$. For both controllers, designed through the proposed methodology:

$$
\begin{gathered}
\sigma_{0}(s)=M_{0} \operatorname{sat}\left(s / M_{0}\right) \\
\sigma_{i j}(s)=M_{i j} \tanh \left(s / M_{i j}\right) \quad \forall(i, j) \in \mathbb{N}_{2} \times \mathbb{N}_{2} \backslash\{(2,2)\} \\
\sigma_{22}(s)= \begin{cases}M_{22}^{-} \tanh \left(s / M_{22}^{-}\right) & \forall s<0 \\
M_{22}^{+} \tanh \left(s / M_{22}^{+}\right) & \forall s \geq 0\end{cases} \\
\sigma_{30}(s)=M_{30} \operatorname{sat}\left(s / M_{30}\right) \\
\sigma_{31}(s)=\operatorname{sign}(s) \min \left\{|s|^{\beta_{31}}, M_{31}\right\} \\
\sigma_{m n}(s)=\sigma_{u}\left(s ; \beta_{m n}\right) \quad \forall(m, n) \in\{4,5,6\} \times\{1,2\}
\end{gathered}
$$

For controller 1:

$$
\sigma_{32}(s)=\sigma_{b}\left(s ; \beta_{32}, L_{32}, M_{32}\right) \quad \text { and } \quad \sigma_{33}(s)=\sigma_{u}\left(s ; \beta_{33}\right)
$$

For controller 2:

$$
\sigma_{32}(s)=\sigma_{u}\left(s ; \beta_{32}\right) \quad \text { and } \quad \sigma_{33}(s)=\sigma_{b}\left(s ; \beta_{33}, L_{33}, M_{33}\right)
$$

\section{ACKNOWLEDGEMENTS}

This work was carried out in the framework of the Labex MS2T, which was funded by the French Government, through the program "Investments for the future" managed by the National Agency for Research (Reference ANR-11-IDEX-0004-02). The third author is financed by the European Regional Development Fund. European Union is investing in your future.

\section{NOTES ON CONTRIBUTORS}

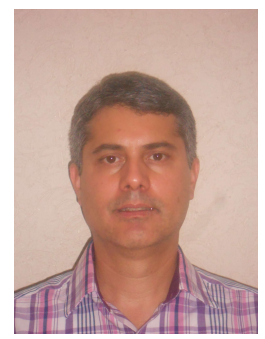

Arturo Zavala-Río received his B.S. degree in Electronic Systems Engineering and M.S. degree in Control Engineering from the Instituto Tecnológico y de Estudios Superiores de Monterrey, Mexico, in 1989 and 1992 respectively, and his D.E.A. and Ph.D. degrees in Automatic Control from the Institut National Politechnique de Grenoble, France, in 1994 and 1997 respectively. He held professor-researcher positions at Universidad Autónoma de Querétaro (1999-2000), Mexico, and Universidad Autónoma de San Luis Potosí (2001), Mexico. He has been visiting researcher at Mechanical Engineering Laboratory (1998), Japan, and Université de Technologie de Compiègne (2001-2002; 2013-2014), France. Since 2002, he is a full-time researcher at the Instituto Potosino de 
Investigación Científica y Tecnológica, Mexico. His research topics focus on the modelling, analysis, and control of nonlinear systems.

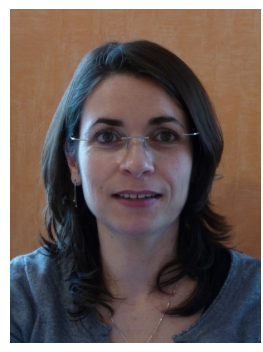

Isabelle Fantoni received the $\mathrm{PhD}$ degree, with the European label, in Non-linear Control for Underactuated Mechanical Systems, in 2000 from the Université de Technologie de Compiègne, in France. Since October 2001, she is a permanent Researcher at Heudiasyc laboratory, UTC, in Compiègne, France, employed by the French National Foundation for Scientific Research (CNRS) and CNRS Research Director since October 2013. Her research interests include non-linear control, modelling and control for Unmanned Aerial Vehicles (UAVs), fault-tolerant control for UAVs, vision for navigation of aerial vehicles, cooperation of UAVs, heterogeneous robotic systems in cooperation.

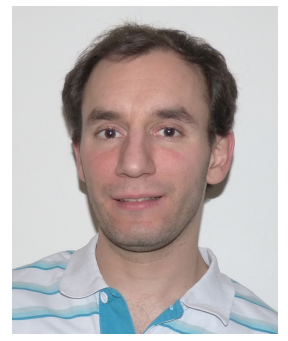

Guillaume Sanahuja has done his $\mathrm{PhD}$ thesis at Heudiasyc laboratory, Université de Technologie de Compiègne, in 2010 on the topic of vision for UAVs. He is now working in the same laboratory as a research engineer. His work is related to the French project Equipex (equipment of excellence) ROBOTEX, which aim is to equip Heudiasyc and its partners with robotic platforms. His function is to design and develop embedded architectures at both software and hardware levels. His topics of interests are embedded systems, embedded vision, non-linear control.

\section{REFERENCES}

[Aeyels and de Leenheer (2002)] Aeyels, D., and de Leenheer, P. (2002), 'Extension of the Perron-Frobenious theorem to homogeneous systems', SIAM Journal of Control and Optimization, 41, 563-582.

[Ailon (2010)] Ailon, A. (2010), 'Simple tracking controllers for autonomous VTOL aircraft with bounded inputs', IEEE Transactions on Automatic Control, $55,737-743$.

[Apostol (1974)] Apostol, T.M. (1974), Mathematical Analysis, 2nd edn, Reading, MA: Addison-Wesley.

[Bacciotti and Rosier (2005)] Bacciotti, A., and Rosier, L. (2005), Liapunov Functions and Stability in Control Theory, 2nd edn, Berlin: Springer.

[Bhat and Bernstein (2000)] Bhat, S.P., and Bernstein, D.S. (2000), 'Finite-time stability of continuous autonomous systems', SIAM Journal on Control and Optimization, 38, 751-766.

[Bhat and Bernstein (2005)] Bhat, S.P., and Bernstein, D.S. (2005), 'Geometric homogeneity with applications to finite-time stability', Mathematics of Control, Signals, and Systems, 17, 101-127.

[Castillo et al.(2004)] Castillo, P., Dzul, A., and Lozano, R. (2004), 'Real-time stabilization and tracking of a four-rotor mini rotorcraft', IEEE Transactions on Control Systems Technology, 12, 510-516.

[Chemori and Marchand (2008)] Chemori, A., and Marchand, N. (2008), 'A prediction-based nonlinear controller for stabilization of a non-minimum phase PVTOL aircraft', International Journal of Robust and Nonlinear Control, 18, 876-889.

[Consolini et al.(2010)] Consolini, L., Maggiore, M., Nielsen, C., and Tosques, M. (2010), 'Path following for the PVTOL aircraft', Automatica, 46, 12841296.

[Consolini and Tosques (2007)] Consolini, L., and Tosques, M. (2007), 'On the VTOL exact tracking with bounded internal dynamics via a Poincaré map approach', IEEE Transactions on Automatic Control, 52, 1757-1762.

[Do et al.(2003)] Do, K.D., Jiang, Z.P., and Pan, J. (2003), 'On the global tracking control of a VTOL aircraft without velocity measurements', IEEE Transactions on Automatic Control, 48, 2212-2217.

[Fantoni and Lozano (2002)] Fantoni, I., and Lozano, R. (2002), Nonlinear Control for Underactuated Mechanical Systems, London: Springer.

[Frye et al.(2010)] Frye, M.T., Ding, S., Qian, C., and Li, S. (2010), 'Fast convergent observer design for output feedback stabilisation of a planar vertical takeoff and landing aircraft', IET Control Theory and Applications, 4, 690-700.

[Haimo (1986)] Haimo, V.T. (1986), 'Finite time controllers', SIAM Journal on Control and Optimization, 24, 760-770.

[Hauser et al.(1992)] Hauser, J., Sastry, S., and Meyer, G. (1992), 'Nonlinear control design for slightly nonminimum phase systems: Application to V/STOL aircraft', Automatica, 28, 665-679.

[Hong (2002)] Hong, Y. (2002), 'Finite-time stabilization and stabilizability of a class of controllable systems', Systems \& Control Letters, 46, 231-236.

[Hong et al.(2001)] Hong, Y., Huang, J., and Xu, Y. (2001), 'On an output feedback finite-time stabilization problem', IEEE Transactions on Automatic Control, 46, 305-309.

[Khalil (2002)] Khalil, H.K. (2002), Nonlinear Systems, 3rd edn, Upper Saddle River, NJ: Prentice Hall.

[Lin et al.(1999)] Lin, F., Zhang, W., and Brandt, R.D. (1999), 'Robust hovering control of a PVTOL aircraft', IEEE Transactions on Control Systems Technology, 7, 343-351.

[Liu and Yu (2013)] Liu, Y., and Yu, H. (2013), 'A survey of underactuated mechanical systems', IET Control Theory and Applications, 7, 921-935.

[Lozano et al.(2004)] Lozano, R., Castillo, P., and Dzul, A. (2004), 'Global stabilization of the PVTOL: real-time application to a mini-aircraft', International Journal of Control, 77, 735-740.

[Lopez-Araujo et al.(2010)] López-Araujo, D.J., Zavala-Río, A., Fantoni, I., Salazar, S., and Lozano, R. (2010), 'Global stabilisation of a PVTOL aircraft with lateral force coupling and bounded inputs', International Journal of Control, 83, 1427-1441.

[Marconi et al.(2002)] Marconi, L., Isidori, A., and Serrani, A. (2002), 'Autonomous vertical landing on an oscillating platform: An internal-model based approach', Automatica, 38, 21-32.

[Martin et al.(1996)] Martin, P., Devasia, S., and Paden, B. (1996), 'A different look at output tracking: Control of a VTOL aircraft', Automatica, 32 , $101-107$.

[Michel et al.(2008)] Michel, A.N., Hou, L., and Liu, D. (2008), Stability of Dynamical Systems, Boston: Birkhäuser.

[Olfati-Saber (2002)] Olfati-Saber, R. (2002), 'Global configuration stabilization for the VTOL aircraft with strong input coupling', IEEE Transactions on Automatic Control, 47, 1949-1952.

[Saeed and Gratton (2010)] Saeed, B., and Gratton, G.B. (2010), 'An evaluation of the historical issues associated with acieving non-helicopter V/STOL capability and the search for the flying car', The Aeronautical Journal, 114, 91-102. 
[Sanahuja et al.(2010)] Sanahuja, G., Castillo-García, P., and Sanchez, A. (2010), 'Stabilization of $n$ integrators in cascade with bounded input with experimental application to a VTOL laboratory system', International Journal of Robust and Nonlinear Control, $20,1129-1139$.

[Setlur et al.(2001)] Setlur, P., Dawson, D., Fang, Y., and Costic, B. (2001), 'Nonlinear tracking control of the VTOL aircraft', in 40th IEEE Conference on Decision and Control, Orlando, FL, 4-7 December 2001, pp. 4592-4597.

[Su and Lin (2011)] Su, S., and Lin, Y. (2011), 'Robust output tracking control of a class of non-minimum phase systems and application to VTOL aircraft', International Journal of Control, 84, 1858-1872.

[Su and Lin (2013)] Su, S., and Lin, Y. (2013), 'Output tracking control for a velocity-sensorless VTOL aircraft with measurement delays', International Journal of Systems Science, DOI: 10.1080/00207721.2013.801091.

[Teel (1992)] Teel, A.R. (1992), 'Global stabilization and restricted tracking for multiple integrators with bounded controls', Systems \& Control Letters, 18, $165-171$.

[Turker, Gorgun and Canserver (2012)] Turker, T., Gorgun, H., and Cansever, G. (2012), 'Stabilization of uncoupled PVTOL aircraft based on a Lyapunov function', Transactions of the Institute of Measurement and Control, 34, 578-584.

[Turker, Olfaz, Gorgun and Canserver (2012)] Turker, T., Oflaz, T., Gorgun, H., and Cansever, G. (2012), 'A stabilizing controler for PVTOL aircraft', in 2012 American Control Conference, Montréal, Canada, 27-29 June 2012, pp. 909-913.

[Wang et al.(2009)] Wang, X., Liu, J., and Cai, K.Y. (2009), 'Tracking control for a velocity-sensorless VTOP aricraft with delayed outputs', Automatica, 45, 2876-2882.

[Wang et al.(2010)] Wang, X., Liu, J., and Cai, K.Y. (2010), 'Tracking control for VTOP aricraft with disabled IMUs', International Journal of Systems Science, 41, 1231-1239.

[Wang et al.(2008)] Wang, X., Yang, G., and Xu, W. (2008), 'Output tracking for nonlinear non-minimum phase systems and application to PVTOL aircraft', International Journal of Systems Science, 39, 29-42.

[Wood and Cazzolato (2007)] Wood, R., and Cazzolato, B. (2007), 'An alternative nonlinear control law for the global stabilization of the PVTOL vehicle', IEEE Transactions on Automatic Control, 52, 1282-1287.

[Ye et al.(2007)] Ye, H., Wang, H., and Wang, H. (2007), 'Stabilization of a PVTOL aircraft and an inertia wheel pendulum using saturation technique', IEEE Transactions on Control Systems Technology, 15, 1143-1150.

[Zavala-Río and Fantoni (2014)] Zavala-Río, A., and Fantoni, I. (2014), 'Global finite-time stability characterized through a local notion of homogeneity', IEEE Transactions on Automatic Control, 59, 471-477.

[Zavala-Río et al.(2003)] Zavala-Río, A., Fantoni, I., and Lozano, R. (2003), 'Global stabilization of a PVTOL aircraft model with bounded inputs', International Journal of Control, 76, 1833-1844. 